\title{
CMS Collaboration
}

T.K. Aarrestad ${ }^{224}$, D. Abbaneo ${ }^{28}$, G. Abbiendi ${ }^{91}$, M. Abbrescia ${ }^{90,13}$, A.A. Abdelalim ${ }^{86,238,1}$, S. Abdullin ${ }^{70}$, A. Abdulsalam ${ }^{19}$, S. Abu Zeid ${ }^{231}$,

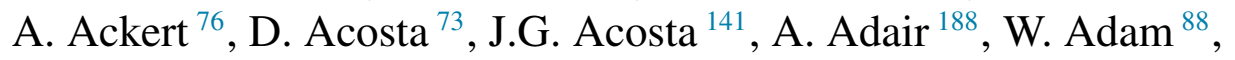
J.R. Adams ${ }^{76}$, M.R. Adams ${ }^{105}$, T. Adams ${ }^{76}$, A. Adiguzel ${ }^{53}$, P. Adzic ${ }^{68,16}$, S. Afanasiev ${ }^{116}$, A. Agapitos ${ }^{9}$, R. Aggleton ${ }^{24}$, J.-L. Agram ${ }^{84,178}$, A. Ahmad ${ }^{148}$, M. Ahmad ${ }^{148}$, M. Ahmad ${ }^{87}$, I. Ahmed ${ }^{146}$, S. Ahuja ${ }^{63}$, M. Akbiyik ${ }^{66}$, N. Akchurin ${ }^{218}$, B. Akgun ${ }^{188}$, I.V. Akin ${ }^{137}$, C. Albajar ${ }^{11}$, M. Albrow ${ }^{70}$, J. Alcaraz Maestre ${ }^{44}$, W.L. Aldá Júnior ${ }^{42}$, M. Aldaya Martin ${ }^{59}$, S. Alderweireldt ${ }^{6}$, A. Aleksandrov ${ }^{161}$, J. Alexander ${ }^{52}$, J. Alimena ${ }^{26}$, L. Alunni Solestizi ${ }^{99,172}$, G. Alverson ${ }^{156}$, F.L. Alves ${ }^{42}$, G.A. Alves ${ }^{42}$, M. Alyari ${ }^{209}$, N. Amapane ${ }^{102,220}$, C. Amsler ${ }^{5,224}$, G. Anagnostou ${ }^{162}$, D. Anderson ${ }^{31}$, I. Anderson ${ }^{115}$, J. Andrea ${ }^{178}$, V. Andreev ${ }^{166}$, Yu. Andreev ${ }^{160}$, M.B. Andrews ${ }^{37}$, K. Androsov ${ }^{210,100}$, C. Anelli ${ }^{134}$, L. Antonelli ${ }^{163}$, I. Antropov ${ }^{124}$, Z. Antunovic ${ }^{205}$, L. Apanasevich ${ }^{105}$, G. Apollinari ${ }^{70}$, E. Appelt ${ }^{226}$, A. Apresyan ${ }^{31}$, A. Apyan ${ }^{135}$, D. Arcaro ${ }^{22}$, R. Arcidiacono ${ }^{28,102,174}$, M.W. Arenton ${ }^{229}$, S. Argiro ${ }^{102,220}$, M. Arneodo ${ }^{102,174}$, S. Arora ${ }^{193}$, B. Asavapibhop ${ }^{48}$, C. Asawatangtrakuldee ${ }^{207}$, E. Asilar ${ }^{88}$, I. Asin ${ }^{59}$, A. Askew ${ }^{76}$, M. Ata ${ }^{185}$, A. Attikis ${ }^{54}$, A. Aubin ${ }^{178}$, E. Auffray ${ }^{28}$, C. Autermann ${ }^{184}$, G. Auzinger ${ }^{28}$, E. Avdeeva ${ }^{155}$, P. Avery ${ }^{73}$, A. Avetisyan ${ }^{22}$, C. Avila ${ }^{130}$, A. Awad ${ }^{1}$, M. Azarkin ${ }^{151,166}$, I. Azhgirey ${ }^{208}$, T. Aziz ${ }^{212}$, P. Azzi ${ }^{28,97}$, V. Azzolini ${ }^{37}$, P. Azzurri ${ }^{28,100}$, M.M. Baarmand ${ }^{74}$, M. Baber ${ }^{106}$, N. Bacchetta ${ }^{97}$, F. Bachmair ${ }^{169}$, M. Bachtis ${ }^{28}$, A. Baden ${ }^{134}$,

S. Baffioni ${ }^{124}$, G. Bagliesi ${ }^{100}$, P. Baillon ${ }^{28}$, R. Bainbridge ${ }^{106}$, H. Bakhshiansohi ${ }^{187}$, A.H. Ball ${ }^{28}$, F. Ball ${ }^{24}$, Y. Ban ${ }^{207}$, S. Banerjee ${ }^{70}$, S. Banerjee ${ }^{212}$, L. Bäni ${ }^{169}$, S. Bansal ${ }^{168}$, G. Barbagli ${ }^{93}$, E. Barberis ${ }^{156}$,

R. Barbieri ${ }^{135}$, P. Bargassa ${ }^{126}$, P. Baringer ${ }^{119}$, A. Barker ${ }^{193}$, V.E. Barnes ${ }^{183}$, B.A. Barnett ${ }^{115}$, D. Barney ${ }^{28}$, O. Baron ${ }^{134}$, L. Barone ${ }^{101,190}$, P. Barria ${ }^{129}$, R. Bartek ${ }^{153}$, C. Barth ${ }^{66}$, M. Bartók ${ }^{234,56}$, 
N. Bartosik ${ }^{59}$, S. Basegmez ${ }^{39}$, A. Baskakov ${ }^{202}$, C. Battilana ${ }^{28}$,

A. Baty ${ }^{135}$, L.A.T. Bauerdick ${ }^{70}$, D. Baumgartel ${ }^{156}$, C. Baus ${ }^{66}$, I. Bayshev ${ }^{208}$, A. Bean ${ }^{119}$, S. Beauceron ${ }^{131}$, F. Beaudette ${ }^{124}$, L. Beck ${ }^{24}$, K. Beernaert ${ }^{81}$, H. Behnamian ${ }^{187}$, O. Behnke ${ }^{59}$, U. Behrens ${ }^{59}$, S. Bein ${ }^{76}$,

C. Beirão Da Cruz E Silva ${ }^{126}$, E. Belchior Batista Das Chagas ${ }^{62}$, S. Belforte ${ }^{103}$, N. Beliy ${ }^{142}$, D.A. Belknap ${ }^{235}$, A.J. Bell ${ }^{59}$, R. Bellan ${ }^{102,220}$, A. Belloni ${ }^{134}$, C. Beluffi ${ }^{178,39}$, A. Belyaev ${ }^{202}$, A. Belyaev ${ }^{196,194}$, A. Benaglia ${ }^{28}$, L. Benato ${ }^{97,167}$, G. Bencze ${ }^{234}$, J. Bendavid ${ }^{28}$, D. Benedetti ${ }^{183}$, G. Benelli ${ }^{119}$, L. Benhabib ${ }^{28}$, N. Beni ${ }^{159}$, J.F. Benitez ${ }^{28}$, L. Benucci ${ }^{81}$, L. Benussi ${ }^{89}$, A.C. Benvenuti ${ }^{91}$, S. Beranek ${ }^{184}$, A. Beretvas ${ }^{70}$, T. Bergauer ${ }^{88}$, J. Berger ${ }^{66}$, S.B. Beri ${ }^{168}$, C.A. Bernardes ${ }^{69}$, J. Bernardini ${ }^{100}$, C. Bernet ${ }^{131}$, G.M. Berruti ${ }^{28}$, D. Berry ${ }^{105}$, E. Berry ${ }^{26}$, J. Berryhill ${ }^{70}$, W. Bertl ${ }^{170}$, M. Besancon ${ }^{55}$, B. Betchart ${ }^{189}$, R.R. Betts ${ }^{105}$, A. Bhardwaj ${ }^{57}$, P.C. Bhat ${ }^{70}$, V. Bhatnagar ${ }^{168}$, S. Bhattacharya ${ }^{195}$, S. Bhattacharya ${ }^{26}$, V. Bhopatkar ${ }^{74}$, S. Bhowmik ${ }^{230,212}$, H. Bialkowska ${ }^{145}$, J.G. Bian ${ }^{87}$, L. Bianchini ${ }^{169}$, S. Bianco ${ }^{89}$, M. Biasini ${ }^{99,172}$, K. Bierwagen ${ }^{135}$, C. Biino ${ }^{102}$, G.M. Bilei ${ }^{99}$, B. Bilin ${ }^{137}$, B. Bilki ${ }^{7,111}$, S. Bilmis ${ }^{137}$, S. Bitioukov ${ }^{208}$, F. Blekman ${ }^{231}$, V. Blobel ${ }^{83}$, D. Bloch ${ }^{178}$, P. Bloch ${ }^{28}$, K. Bloom ${ }^{155}$, M. Bluj ${ }^{145}$, B. Blumenfeld ${ }^{115}$, T. Boccali ${ }^{100}$, A. Bocci ${ }^{28}$, J. Bochenek ${ }^{76}$, A. Bodek ${ }^{189}$, M. Bodlak ${ }^{45}$, B. Boimska ${ }^{145}$, A. Boletti ${ }^{97,167}$, G. Bolla ${ }^{70}$, D. Bonacorsi ${ }^{91,21}$, A. Bonato ${ }^{28}$, O. Bondu ${ }^{39}$, E. Boos ${ }^{202}$, A. Bornheim ${ }^{31}$, K. Borras ${ }^{185,59}$, P. Bortignon ${ }^{73}$, D. Bortoletto ${ }^{183}$, A. Borzou ${ }^{15}, \mathrm{~S}$. Bose ${ }^{155}$, T. Bose ${ }^{22}$, C. Böser ${ }^{66}$, C. Botta ${ }^{28}$, G. Boudoul ${ }^{131}$, O. Bouhali ${ }^{217,216}$, D. Bourilkov ${ }^{73}$, E. Bouvier ${ }^{131}$, J. Bradmiller-Feld ${ }^{35}$, A. Braghieri ${ }^{98}$, S. Braibant-Giacomelli ${ }^{91,21}$, A. Branca ${ }^{97,167}$, J. Brandstetter ${ }^{88}$, S. Brandt ${ }^{135}$, J.G. Branson ${ }^{34}$, R. Breedon ${ }^{30}$, G. Breto ${ }^{30}$, H. Breuker ${ }^{28}$, C. Brew ${ }^{194}$, L. Brianza ${ }^{95,138}$, L. Brigliadori ${ }^{91,21}$, V. Brigljevic ${ }^{191}$, A. Brinkerhoff ${ }^{158}$, J. Brinson ${ }^{163}$, L. Brito ${ }^{42}$, J.A. Brochero Cifuentes ${ }^{46}$, S. Brochet ${ }^{39}$, M. Brodski ${ }^{185}$, J.-M. Brom ${ }^{178}$, G. Brona ${ }^{65}$, E. Brondolin ${ }^{88}$, J.J. Brooke ${ }^{24}$, R.M. Brown ${ }^{194}$, H. Brun ${ }^{129}$, C. Bruner ${ }^{119}$, G. Bruno ${ }^{39}$, O. Buchmuller ${ }^{106}$, I. Bucinskaite ${ }^{105}$, A. Bundock ${ }^{106}$, P. Bunin ${ }^{116}$, K. Bunkowski ${ }^{65}$, J. Bunn ${ }^{31}$, S. Buontempo ${ }^{96}$, A. Burgmeier ${ }^{59}$, K. Burkett ${ }^{70}$, K. Burt ${ }^{33}$, D. Burton ${ }^{106}$, P. Busson ${ }^{124}$, W. Busza ${ }^{135}$, J.N. Butler ${ }^{70}$, P.H. Butler ${ }^{36}$, M. Buttignol ${ }^{178}$, E. Butz ${ }^{66}$, A. Bylinkin ${ }^{151}$, B. Bylsma ${ }^{163}$, A. Byszuk ${ }^{232,65}$, A. Cabrera ${ }^{130}$, I.J. Cabrillo ${ }^{72}$, L. Cadamuro ${ }^{124}$, C. Caillol ${ }^{129}$, A. Cakir ${ }^{113}$, C. Calabria ${ }^{90,13}$, 
A. Calamba ${ }^{37}$, A. Calderon ${ }^{72}$, M. Calderon De La Barca Sanchez ${ }^{30}$, I.A. Cali ${ }^{135}$, K. Call ${ }^{15}$, L. Calligaris ${ }^{194}$, B. Calpas ${ }^{149}$, V. Calvelli ${ }^{94,79}$, B. Calvert ${ }^{134}$, E. Calvo ${ }^{44}$, L. Caminada ${ }^{224}$, C. Campagnari ${ }^{35}$, R. Campanini ${ }^{91,21}$, A. Campbell ${ }^{59}$, T. Camporesi ${ }^{28}$, V. Candelise ${ }^{28,103,223}$, M.F. Canelli ${ }^{224}$, K. Cankocak ${ }^{113}$, P. Capiluppi ${ }^{91,21}$, G. Cappello ${ }^{92}$,

C. Caputo ${ }^{90,13}$, M. Cardaci ${ }^{144}$, D. Carlsmith ${ }^{235}$, B. Carlson ${ }^{37}$, A. Carnes ${ }^{73}$, C.A. Carrillo Montoya ${ }^{131}$, S. Carrillo Moreno ${ }^{104}$, N. Cartiglia ${ }^{102}$, W. Carvalho ${ }^{62}$, M. Carver ${ }^{73}$, B. Casal ${ }^{169}$, M. Casarsa ${ }^{103}$, S. Casasso ${ }^{106}$, E. Casimiro Linares ${ }^{43}$, R. Castaldi ${ }^{100}$,

A. Castaneda Hernandez ${ }^{217,216}$, R. Castello ${ }^{28}$, J.R. Castiñeiras De Saa ${ }^{72}$, H. Castilla-Valdez ${ }^{43}$, A. Castro ${ }^{91,21}$, A. Caudron ${ }^{39}$, F. Cavallari ${ }^{101}$, F.R. Cavallo ${ }^{91}$, N. Cavallo ${ }^{96,14}$, R. Cavanaugh ${ }^{105}$, L. Ceard ${ }^{39}$, A. Celik ${ }^{216}$, M. Centis Vignali ${ }^{83}$, M. Cepeda ${ }^{235}$, G.B. Cerati ${ }^{34}$, S. Cerci ${ }^{2,53}$, G. Cerminara ${ }^{28}$, M. Cerrada ${ }^{44}$, E.C. Chabert ${ }^{178}$, M. Chamizo Llatas ${ }^{44}$, P. Chang ${ }^{153}$, Y.H. Chang ${ }^{153}$, Y.W. Chang ${ }^{153}$, N. Chanon ${ }^{178}$, Y. Chao ${ }^{153}$, L.F. Chaparro Sierra ${ }^{130}$, E. Chapon ${ }^{124}$, O. Charaf ${ }^{4}$, C. Charlot ${ }^{124}$, M. Chasco ${ }^{156}$, A. Chatterjee ${ }^{52}$, K. Chatterjee ${ }^{195}$, R.M. Chatterjee ${ }^{212}$, S. Chauhan ${ }^{108}$, S. Chauhan ${ }^{30}$, J. Chaves ${ }^{52}$, R. Chawla ${ }^{168}$, G.M. Chen ${ }^{87}$, H.S. Chen ${ }^{87}$, K.F. Chen ${ }^{153}$, K.H. Chen ${ }^{144}$, M. Chen ${ }^{87}$, P.H. Chen ${ }^{153}$, Y. Chen ${ }^{31}$, Z. Chen ${ }^{188}$, T. Cheng ${ }^{87}$, V. Cherepanov ${ }^{186}$, M. Chertok ${ }^{30}$, H.W.K. Cheung ${ }^{70}$, S.S. Chhibra ${ }^{91,21}$, R. Chierici ${ }^{131}$, J. Chinellato ${ }^{64,62}$, V. Chiochia ${ }^{224}$, M. Chiorboli ${ }^{92,38}$, F. Chlebana ${ }^{70}$, M. Choi ${ }^{201}$, S. Choi ${ }^{122}$, Y. Choi ${ }^{211}$, J.P. Chou ${ }^{193}$, B.C. Choudhary ${ }^{57}$, S. Choudhury ${ }^{107,59}$, J. Chu ${ }^{52}$, R. Chudasama ${ }^{19}$, T. Chwalek ${ }^{66}$, D. Ciangottini ${ }^{28,99,172}$, D. Cieri ${ }^{194}$,

S. Cihangir ${ }^{70}$, A. Cimmino ${ }^{81}$, M.A. Ciocci ${ }^{210,100}$, P. Cirkovic ${ }^{16}$, M. Citron ${ }^{106}$, S. Cittolin ${ }^{34}$, V. Ciulli ${ }^{93,71}$, C. Civinini ${ }^{93}$, D.R. Claes ${ }^{155}$, R. Clare ${ }^{33}$, W. Clarida ${ }^{111}$, C. Clarke ${ }^{233}$, E. Clement ${ }^{24}$, B. Clerbaux ${ }^{129}$, D.J.A. Cockerill ${ }^{194}$, G. Codispoti ${ }^{91,21}$, S. Colafranceschi ${ }^{67,74}$, A. Colaleo ${ }^{90}$, J.E. Cole ${ }^{27}$, N. Colino ${ }^{44}$, C. Collard ${ }^{178}$, D. Colling ${ }^{106}$, F. Colombo ${ }^{66}$, D. Contardo ${ }^{131}$, E. Conte ${ }^{84,178}$, C. Contreras-Campana ${ }^{193}$, E. Contreras-Campana ${ }^{193}$, J. Conway ${ }^{30}$, R. Conway ${ }^{30}$, S.I. Cooper ${ }^{4}$, T. Cornelis ${ }^{6}$, L. Corpe ${ }^{106}$, M. Correa Martins Junior ${ }^{42}$, F. Cossutti ${ }^{103}$, M. Costa ${ }^{102,220}$, S. Costa ${ }^{92,38}$, F. Costanza ${ }^{59}$, X. Coubez ${ }^{178}$, F. Couderc ${ }^{55}$, J.A. Coughlan ${ }^{194}$, B. Courbon ${ }^{131}$, R. Cousins ${ }^{32}$, R. Covarelli ${ }^{102,220}$, C. Cowden ${ }^{218}$, B. Cox $^{229}$, P.T. Cox ${ }^{30}$, D. Creanza ${ }^{90,179}$, N. Cripps ${ }^{106}$, L. Cristella ${ }^{90,13}$, S. Crucy ${ }^{81}$, J. Cuevas ${ }^{164}$, M. Cuffiani ${ }^{91,21}$, J.P. Cumalat ${ }^{50}$, D. Curry ${ }^{73}$, D. Cussans ${ }^{24}$, A. Custódio ${ }^{62}$, D. Cutts ${ }^{26}$, 
S. Czellar ${ }^{159}$, R.T. D'Agnolo ${ }^{34}$, R. D'Alessandro ${ }^{93,71}$, M. D'Alfonso ${ }^{28}$, J. D'Hondt ${ }^{231}$, E.M. Da Costa ${ }^{62}$, G.G. Da Silveira ${ }^{39}$, A. Dabrowski ${ }^{28}$, N. Daci ${ }^{231}$, B. Dahmes ${ }^{140}$, T. Dahms ${ }^{124}$, M. Dalchenko ${ }^{216}$, M. Dall'Osso ${ }^{28,97,167}$, G.M. Dallavalle ${ }^{91}$, J. Damgov ${ }^{218}$, V. Daponte ${ }^{28}$, S. Das ${ }^{73}$, G. Daskalakis ${ }^{162}$, S. Dasu ${ }^{235}$, P. Dauncey ${ }^{106}$, A. David ${ }^{28}$, G. Davies ${ }^{106}$, O. Davignon ${ }^{124}$, A. De Roeck ${ }^{28}$, A. De Souza Santos ${ }^{69}$, A. De Wit ${ }^{106}$, A. De Cosa ${ }^{224}$, B. De La Cruz ${ }^{44}$, C. De Oliveira Martins ${ }^{62}$, D. De Jesus Damiao ${ }^{62}$, E. De La Cruz-Burelo ${ }^{43}$, E.A. De Wolf ${ }^{6}$, F. De Guio ${ }^{28}$, G. De Lentdecker ${ }^{129}$, I. De Bruyn ${ }^{231}$, M. De Gruttola ${ }^{28}$, M. De Mattia ${ }^{216}$, M. De Palma ${ }^{90,13}$, N. De Filippis ${ }^{90,179}$, P. De Castro Manzano ${ }^{72}$, S. De Visscher ${ }^{28}$, W. De Boer ${ }^{66}$, A. Degano ${ }^{102,220}$, K. Deiters ${ }^{170}$, M. Dejardin ${ }^{55}$, D. Del Re ${ }^{28,101,190}$, C. Delaere ${ }^{39}$, A.G. Delannoy ${ }^{226}$, A. Delgado ${ }^{216}$, A. Delgado Peris ${ }^{44}$, R. Dell'Orso ${ }^{100}$, G. Della Ricca ${ }^{103,223}$, M. Della Negra ${ }^{106}$, N. Demaria ${ }^{102}$, R. Demina ${ }^{189}$, Z. Demiragli ${ }^{135}$, Z.S. Demiroglu ${ }^{53}$, D. Denegri ${ }^{55}$, P. Depasse ${ }^{131}$, M. Derdzinski ${ }^{34}$, A. Dermenev ${ }^{160}$, K. Deroover ${ }^{231}$, A. Descroix ${ }^{66}$, N. Dev ${ }^{158}$, R.K. Dewanjee ${ }^{212}$, S. Dey ${ }^{195}$, N. Dhingra ${ }^{26}$,

A. Di Francesco ${ }^{126}$, A. Di Mattia ${ }^{92}$, E. Di Marco ${ }^{101,190,28}$, L. Di Matteo ${ }^{135}$, S. Di Guida ${ }^{28,96,77}$, B. Diamond ${ }^{76}$, M. Diemoz ${ }^{101}$, A. Dierlamm ${ }^{66}$, C. Dietz ${ }^{153}$, E. Dietz-Laursonn ${ }^{185}$, C. Diez Pardos ${ }^{59}$, S. Dildick ${ }^{216}$, K. Dilsiz ${ }^{111}$, A. Dimitrov ${ }^{203}$, G. D'imperio ${ }^{28,101,190}$, M.E. Dinardo ${ }^{95,138}$, A. Dishaw ${ }^{35}$, G. Dissertori ${ }^{169}$, J. Dittmann ${ }^{15}$, M. Dittmar ${ }^{169}$, S. Dittmer ${ }^{52}$, T.H. Doan ${ }^{144}$, M. Dobson ${ }^{28}$, D. Dobur ${ }^{81}$, L. Dodd ${ }^{235}$, S. Dogra ${ }^{63}$, J. Dolen ${ }^{209}$, G. Dolinska ${ }^{59}$, A. Dominguez ${ }^{155}$, D. Domínguez Vázquez ${ }^{44}, S$. Donato ${ }^{28,100,198}$, M. Donegà ${ }^{169}$, S. Dooling ${ }^{59}$, M. Dordevic ${ }^{28}$, T. Dorigo ${ }^{97}$, T. Dorland ${ }^{59}$, B. Dorney ${ }^{28}$, K. Doroba ${ }^{65}$, C. Dozen ${ }^{53}$, A.R. Draeger ${ }^{83}$, M. Dragicevic ${ }^{88}$, C. Dragoiu ${ }^{218}$, I. Dremin ${ }^{151,166}$, O. Driga ${ }^{180}$, R. Du ${ }^{87}$, J. Duarte ${ }^{31}$, S. Dube ${ }^{108}$, D. Duchardt ${ }^{185}$, V. Dudenas ${ }^{228}$, P.R. Dudero ${ }^{218}$, S. Dugad ${ }^{212}$, D. Duggan ${ }^{28}$, I. Dumanoglu ${ }^{53}$, P. Dunne ${ }^{106}$, M. Dünser ${ }^{28}$, N. Dupont ${ }^{28}$, S. Durgut ${ }^{111}$, S. Duric ${ }^{235}$, L.S. Durkin ${ }^{163}$, D. Dutta ${ }^{19}$, S. Dutta ${ }^{195}$, V. Dutta ${ }^{35}$, G. Eckerlin ${ }^{59}$, K.M. Ecklund ${ }^{188}$, D. Eckstein ${ }^{59}$, M. Edelhoff ${ }^{184}$, P. Eerola ${ }^{58}$, N. Eggert ${ }^{52}$, T. Eichhorn ${ }^{59}$, H. El Mamouni ${ }^{131}$, M. El Sawy ${ }^{18,25,1}$, P. Eller ${ }^{169}$, A. Elliott-Peisert ${ }^{28}$, J. Ellison ${ }^{33}$, P. Elmer ${ }^{180}$, V.D. Elvira ${ }^{70}$, A. Elwood ${ }^{106}$, N. Eminizer ${ }^{115}$, M. Endres ${ }^{185}$, S.C. Eno ${ }^{134}$, V. Epshteyn ${ }^{219}$, R. Erbacher ${ }^{30}$, M. Erdmann ${ }^{185}$, W. Erdmann ${ }^{170}$, Y. Erdogan ${ }^{186}$, S. Erdweg ${ }^{185}$, J. Erfle ${ }^{83}$, 
J. Erö ${ }^{88}$, A. Ershov ${ }^{202}$, A. Escalante Del Valle ${ }^{44}$, T. Esch ${ }^{185}$, Y. Eshaq ${ }^{189}$, M. Esposito ${ }^{96,143}$, S.M. Etesami ${ }^{112,187}$, R. Eusebi ${ }^{216}$, I. Evangelou ${ }^{110}$, A. Evans ${ }^{140}$, O. Evdokimov ${ }^{105}$, P. Everaerts ${ }^{32}$, F. Fabbri ${ }^{91}$, F. Fabbri ${ }^{89}$, B. Fabbro ${ }^{55}$, F. Fabozzi ${ }^{96,14}$, P. Faccioli ${ }^{126}$, A. Fagot ${ }^{81}$, A. Fahim ${ }^{214,187}$, J. Fan ${ }^{131}$, A. Fanfani ${ }^{91,21}$, C. Fangmeier ${ }^{155}$, L. Fanò ${ }^{99,172}$, C. Fantasia ${ }^{22}$, F. Fanzago ${ }^{97}$, C. Farrell ${ }^{32}$, D. Fasanella ${ }^{91,21}$, G. Fasanella ${ }^{129}$, J. Faulkner ${ }^{218}$, J.L. Faure ${ }^{55}$, C. Favaro ${ }^{55}$, D. Favart ${ }^{39}$, L. Favart ${ }^{129}$, J. Fay ${ }^{131}$, G. Fedi ${ }^{28,100,198}$, D. Fehling ${ }^{115}$, M. Felcini ${ }^{49}$, L. Feld ${ }^{184}$, L. Feng ${ }^{115}$, A. Ferapontov ${ }^{26}$, T. Ferbel ${ }^{189}$, D. Ferencek ${ }^{193}$, T. Ferguson ${ }^{37}$, W. Ferguson ${ }^{106}$, C. Fernandez Bedoya ${ }^{44}$, J. Fernandez Menendez ${ }^{164}$, J.P. Fernández Ramos ${ }^{44}$, M. Fernandez ${ }^{72}$, T.R. Fernandez Perez Tomei ${ }^{63}$,

C. Ferraioli ${ }^{134}$, P.G. Ferreira Parracho ${ }^{126}$, F. Ferri ${ }^{55}$, F. Ferro ${ }^{94}$,

R.D. Field ${ }^{73}$, N. Filipovic ${ }^{124}$, L. Finco ${ }^{28,102,220}$, M. Finger Jr. ${ }^{16,45}$, M. Finger ${ }^{116,45}$, S. Fink ${ }^{66}$, A. Finkel ${ }^{140}$, L. Fiore ${ }^{90}$, S. Fiorendi ${ }^{95,138}$, F. Fiori ${ }^{153}$, R. Fischer ${ }^{185}$, I. Fisk ${ }^{70}$, H. Flacher ${ }^{24}$, M. Flechl ${ }^{88}$, J. Flix ${ }^{44}$, A. Florent ${ }^{124}$, C. Florez ${ }^{130}$, G. Flouris ${ }^{110}$, K. Flowers ${ }^{35}$, S. Flowers ${ }^{163}$, G. Flucke ${ }^{59}$, G. Flügge ${ }^{186}$, L. Foà 100,198,*, E. Focardi ${ }^{93,71}$, M. Foerster ${ }^{215}$, S. Folgueras ${ }^{164}$, S. Fonseca De Souza ${ }^{62}$, J.-C. Fontaine ${ }^{84,178}$, W.T. Ford ${ }^{50}$, L. Forthomme ${ }^{39}$, C. Foudas ${ }^{110}$, M.C. Fouz ${ }^{44}$, B. Francis ${ }^{229}$, M. Franco Sevilla ${ }^{35}$, G. Franzoni ${ }^{28}$, J. Freeman ${ }^{70}$, F. Frensch ${ }^{66}$, M. Friedl ${ }^{88}$, R. Friese ${ }^{66}$, T. Frueboes ${ }^{145}$, R. Frühwirth ${ }^{227,88}$, J. Fulcher ${ }^{28}$, W. Funk ${ }^{28}$, I.K. Furic ${ }^{73}$, D. Futyan ${ }^{106}$, S. Gadrat ${ }^{41}$, M. Galanti ${ }^{189}$, M. Gallinaro ${ }^{126}$, E. Gallo ${ }^{83,59}$, C. Galloni ${ }^{224}$, R.P. Gandrajula ${ }^{111}$, S. Ganguly ${ }^{212}$, S. Ganjour ${ }^{55}$, A. Garabedian ${ }^{26}$, J. Garay Garcia ${ }^{59}$, G. Garcia ${ }^{81}$, P. Garcia-Abia ${ }^{44}$, A. Garcia-Bellido ${ }^{189}$, J. Garcia-Ferrero ${ }^{72}$, M. Gardner ${ }^{30}$, R.B. Garg ${ }^{57}$, E. Garutti ${ }^{83}$, J.W. Gary ${ }^{33}$, S. Gascon ${ }^{131}$, D. Gastler ${ }^{22}$, L. Gauthier ${ }^{105}$, M. Gavrilenko ${ }^{116}$, V. Gavrilov ${ }^{219}$, A. Gaz ${ }^{50}$, H. Geenen ${ }^{186}$, P. Geffert ${ }^{35}$, A. Geiser ${ }^{59}$, M. Geisler ${ }^{186}$, D. Gelé ${ }^{178}$, S. Gelli ${ }^{101,190}$, S. Gennai ${ }^{95}$, C. George ${ }^{35}$, J. George ${ }^{209}$, T. Geralis ${ }^{162}$, C.E. Gerber ${ }^{105}$, R. Gerosa ${ }^{95,138}$, Y. Gershtein ${ }^{193}$, F.J.M. Geurts ${ }^{188}$, V.M. Ghete ${ }^{88}$, A. Ghezzi ${ }^{95,138}$, S. Ghosh ${ }^{212}$, P. Giacomelli ${ }^{91}$, V.A. Giakoumopoulou ${ }^{162}$, A. Giammanco ${ }^{149,39}$, A. Giassi ${ }^{100}$, M. Giffels ${ }^{66}$, D. Gigi ${ }^{28}$, A. Gilbert ${ }^{66}$, K. Gill ${ }^{28}$, J. Gilmore ${ }^{216}$, D. Giordano ${ }^{28}$, F. Giordano ${ }^{92,38}$, S. Girgis ${ }^{53}$, M. Girone ${ }^{28}$, A. Givernaud ${ }^{55}$, A. Gizhko ${ }^{59}$, F. Glege ${ }^{28}$, S.V. Gleyzer ${ }^{73}$, I. Glushkov ${ }^{203}$, S. Gninenko ${ }^{160}$, Y. Go ${ }^{122}$, B. Gobbo ${ }^{103}$, N. Godinovic ${ }^{204}$, A. Godshalk ${ }^{209}$, K. Goebel ${ }^{83}$, U. Goerlach ${ }^{178}$, C. Goetzmann ${ }^{178}$, 
J. Goh ${ }^{211}$, G. Gokbulut ${ }^{53}$, R. Goldouzian ${ }^{187}$, J. Goldstein ${ }^{24}$, F. Golf ${ }^{35}$, V. Golovtsov ${ }^{173}$, N. Golubev ${ }^{160}$, I. Golutvin ${ }^{116}$, B. Gomber ${ }^{235}$, B. Gomez Moreno ${ }^{130}$, G. Gomez Ceballos ${ }^{135}$, G. Gomez ${ }^{72}$, J.A. Gomez ${ }^{134}$, J.P. Gomez ${ }^{130}$, M. Goncharov ${ }^{135}$, F. Gonella ${ }^{97}$, D. Gonzalez ${ }^{83}$, I. Gonzalez Caballero ${ }^{164}$, O. Gonzalez Lopez ${ }^{44}$, R. Gonzalez Suarez ${ }^{155}$, S. Gonzi ${ }^{93,71}$, J. Goodell ${ }^{229}$, I. Gorbunov ${ }^{116}$, V. Gori ${ }^{93,71}$, M. Görner ${ }^{83}$, M. Górski ${ }^{145}$, E. Gottschalk ${ }^{70}$, L. Gouskos ${ }^{35}$, M. Gouzevitch ${ }^{131}$, P. Govoni ${ }^{95,138}$, S. Goy Lopez ${ }^{44}$, A. Gozzelino ${ }^{97}$,

C. Grab ${ }^{169}$, J. Gran ${ }^{35}$, C. Grandi ${ }^{91}$, R. Granier de Cassagnac ${ }^{124}$, P. Gras ${ }^{55}$, L. Gray ${ }^{70}$, R. Gray ${ }^{193}$, A. Grebenyuk ${ }^{129}$, D. Green ${ }^{70}$, S. Greene ${ }^{226}$, E.M. Gregores ${ }^{69}$, A. Gribushin ${ }^{202}$, M. Grimes ${ }^{24}$, M.T. Grippo ${ }^{210,100}$, A.V. Gritsan ${ }^{115}$, M. Grothe ${ }^{235}$, U. Grundler ${ }^{153}$, S. Grünendahl ${ }^{70}$, M. Grunewald ${ }^{49}$, B. Grynyov ${ }^{197}$, M. Guchait ${ }^{212}$, A. Gude ${ }^{140}$, R. Guida ${ }^{28}$, L. Guiducci ${ }^{91,21}$, M. Guilbaud ${ }^{188}$, M. Gul ${ }^{81}$, Y. Guler ${ }^{53}$, D. Gulhan ${ }^{135}$, E. Gülmez ${ }^{20}$, S. Gundacker ${ }^{28}$, P. Gunnellini ${ }^{59}$, R. Gupta ${ }^{168}$, E. Gurpinar ${ }^{53}$, A. Gurrola ${ }^{226}$, A. Gurtu ${ }^{121,212}$, L. Gutay ${ }^{183}$, A. Güth ${ }^{185}$, M. Guthoff ${ }^{28}$, O. Gutsche ${ }^{70}$, D. Gyun ${ }^{122}$, J. Haas ${ }^{76}$, R. Hadjiiska ${ }^{161}$, N.J. Hadley ${ }^{134}$, S. Hagopian ${ }^{76}$, V. Hagopian ${ }^{76}$, K.A. Hahn ${ }^{157}$, D. Haitz ${ }^{66}$, C. Hajdu ${ }^{234}$, J. Hakala ${ }^{26}$, E. Halkiadakis ${ }^{193}$, G. Hall ${ }^{106}$, R. Hall-Wilton ${ }^{235}$, J. Haller ${ }^{83}$, G. Hamel de Monchenault ${ }^{55}$, M. Hamer ${ }^{42}$, G.H. Hammad ${ }^{142}$, J. Hammer ${ }^{28}$, J. Han ${ }^{189}$, J. Hanlon ${ }^{70}$, P. Hansen ${ }^{140}$, G. Hanson ${ }^{33}$, J. Hardenbrook ${ }^{180}$, K. Harder ${ }^{194}$, D. Hare ${ }^{70}$, A. Harel ${ }^{189}$, J. Härkönen ${ }^{85}$, S. Harper ${ }^{194}$, R. Harr ${ }^{233}$, C. Harrington ${ }^{209}$, P. Harris ${ }^{28}$, R.M. Harris ${ }^{70}$, A. Hart ${ }^{163}$, C. Hartl ${ }^{88}$, F. Hartmann ${ }^{28,66}$, S. Hasegawa ${ }^{70}$, Q. Hassan ${ }^{148}$, K. Hatakeyama ${ }^{15}$, J. Hauk ${ }^{59}$, J. Hauser ${ }^{32}$,

M. Haytmyradov ${ }^{111}$, A. Hazi ${ }^{234}$, G.P. Heath ${ }^{24}$, H.F. Heath ${ }^{24}$,

T. Hebbeker ${ }^{185}$, P. Hebda ${ }^{180}$, J. Hegeman ${ }^{28}$, C. Heidegger ${ }^{169}$,

C. Heidemann ${ }^{185}$, J. Heilman ${ }^{33}$, S.M. Heindl ${ }^{66}$, U. Heintz ${ }^{26}$,

A. Heister ${ }^{184}$, M. Hempel ${ }^{23,59}$, C. Henderson ${ }^{4}$, C. Hensel $^{42}$, N. Heracleous ${ }^{231}$, I. Heredia-De La Cruz ${ }^{51,43}$, J.M. Hernandez ${ }^{44}$,

A. Hernandez-Almada ${ }^{43}$, M. Herndon ${ }^{235}$, A. Hervé ${ }^{235}$,

S. Hewamanage ${ }^{75}$, D. Hidas ${ }^{193}$, P. Hidas ${ }^{234}$, M. Hildreth ${ }^{158}$, C. Hill ${ }^{163}$, O. Hindrichs ${ }^{189}$, A. Hinzmann ${ }^{224}$, R. Hirosky ${ }^{229}$, J. Hirschauer ${ }^{70}$, D. Hits ${ }^{169}$, P.R. Hobson ${ }^{27}$, F. Hoehle ${ }^{186}$, K. Hoepfner ${ }^{185}$, M. Hoffmann ${ }^{83}$, D.J. Hofman ${ }^{105}$, M. Hohlmann ${ }^{74}$, R.S. Höing ${ }^{83}$, J. Hollar ${ }^{39}$, A. Holzner ${ }^{34}$, B. Hong ${ }^{122}$, H.R. Hoorani ${ }^{148}$, R. Horisberger ${ }^{170}$, N. Hörmann ${ }^{88}$, A. Hortiangtham ${ }^{156}$, D. Horvath ${ }^{159,234}$, I. Hos ${ }^{53}$, 
J. Hoss ${ }^{169}$, W.-S. Hou ${ }^{153}$, T. Hreus ${ }^{224}$, J. Hrubec ${ }^{88}$, Y. Hsiung ${ }^{153}$,

Z. Hu ${ }^{70}$, T. Huang ${ }^{216}$, L.M. Huertas Guativa ${ }^{62}$, E. Hughes ${ }^{193}$, R. Hughes ${ }^{163}$, J. Hugon ${ }^{73}$, U. Husemann ${ }^{66}$, G. Iaselli ${ }^{90,179}$, I. Iashvili ${ }^{209}$, P. Iaydjiev ${ }^{161}$, Z.A. Ibrahim ${ }^{146}$, M. Ignatenko ${ }^{32}$, Y. Iiyama ${ }^{135}$, G. Iles ${ }^{106}$, B. Ille ${ }^{131}$, J. Incandela ${ }^{35}$, Q. Ingram ${ }^{170}$, V. Innocente ${ }^{28}$, G.M. Innocenti ${ }^{135}$, A.O.M. Iorio ${ }^{96,143}$, B. Isildak ${ }^{165,137}$, A. Ivanov ${ }^{120}$, Y. Ivanov ${ }^{173}$, M. Ivova PANEVA ${ }^{33}$, S. Jabeen ${ }^{134}$, J. Jacob ${ }^{24}$, A. Jafari ${ }^{39}$, Sa. Jain ${ }^{195}$, Sh. Jain ${ }^{144}$, P. Jandir ${ }^{33}$, R. Janjam ${ }^{226}$, P. Janot ${ }^{28}$, X. Janssen ${ }^{6}$, P. Jarry ${ }^{55}$, B. Jayatilaka ${ }^{70}$, M. Jeitler ${ }^{227,88}$, F. Jensen ${ }^{50}$, C. Jessop ${ }^{158}$, P. Jez ${ }^{39}$, M.K. Jha ${ }^{183}$, V. Jha ${ }^{19}$, W. Ji ${ }^{163}$, C.H. Jiang ${ }^{87}$, S. Jindariani ${ }^{70}$, M. Jo ${ }^{122}$, W. Johns ${ }^{226}$, A. Johnson ${ }^{50}$, K.F. Johnson ${ }^{76}$, M. Johnson ${ }^{70}$, M. Jones ${ }^{183}$, C. Jorda ${ }^{101}$, M.I. Josa ${ }^{44}$, U. Joshi ${ }^{70}$, A.W. Jung ${ }^{70}$, H. Jung ${ }^{59}$, K. Jung ${ }^{183}$, A. Junkes ${ }^{83}$, A. Juodagalvis ${ }^{228}$, K. Kaadze ${ }^{120}$, V. Kachanov ${ }^{208}$, M. Kadastik ${ }^{149}$, K. Kadija ${ }^{191}$, H.C. Kaestli ${ }^{170}$, J. Kaisen ${ }^{209}$, S. Kalafut ${ }^{140}$, H. Kalakhety ${ }^{74}$, A. Kalinin ${ }^{208}$, A. Kalinowski ${ }^{65}$, A. Kalogeropoulos ${ }^{59}$, A.K. Kalsi ${ }^{168}$,

R. Kamalieddin ${ }^{155}$, A. Kamenev ${ }^{116}$, A. Kaminskiy ${ }^{97,167,222,202,}$ T. Kamon ${ }^{123,216}$, E.E. Kangal ${ }^{136,53}$, K. Kanishchev ${ }^{97,221}$, S.C. Kao ${ }^{140}$, S. Kaplan ${ }^{193}$, O. Karacheban ${ }^{23,59}$, J. Karancsi ${ }^{56,159}$, G. Karapinar ${ }^{114,137}$, G. Karapostoli ${ }^{129}$, P.E. Karchin ${ }^{233}$, B. Kargoll ${ }^{186}$, V. Karimäki ${ }^{85}$, V. Karjavin ${ }^{116}$, D.J. Karmgard ${ }^{158}$, A. Karneyeu ${ }^{160}$, M. Kasemann ${ }^{59}$, G. Kasieczka ${ }^{169}$, I. Katkov ${ }^{202,66}$, P. Katsas ${ }^{59}$, A. Kaur ${ }^{168}$, M. Kaur ${ }^{168}$, M. Kaya ${ }^{133,20}$, O. Kaya ${ }^{117,20}$, A. Kayis Topaksu ${ }^{53}$, M. Kazana ${ }^{145}$, J. Keaveney ${ }^{231}$, N. Kellams ${ }^{158}$, J. Keller ${ }^{155}$, R. Kelley ${ }^{34}$, R.G. Kellogg ${ }^{134}$, E. Kennedy ${ }^{33}$, R.P. Kenny III ${ }^{119}$, M. Kenzie ${ }^{106}$, S. Kesisoglou ${ }^{9}$, V. Khachatryan ${ }^{236}$, M. Khakzad ${ }^{187}$, S. Khalil ${ }^{120}$, A. Khan ${ }^{27}$, W.A. Khan ${ }^{148}$, A. Kharchilava ${ }^{209}$, A. Khatiwada ${ }^{76}$, V. Khristenko ${ }^{111}$, A. Khukhunaishvili ${ }^{189}$, R. Khurana ${ }^{144}$, T. Khurshid ${ }^{148}$, B. Kiani ${ }^{102,220}$, M.K. Kiesel ${ }^{184}$, J. Kieseler ${ }^{59}$, B. Kilminster ${ }^{224}$, D. Kim ${ }^{211}$, D.H. Kim ${ }^{123}$, G.N. Kim ${ }^{123}$, H. Kim ${ }^{46}$, H. Kim ${ }^{122}$, H. Kim ${ }^{201}$, J.H. Kim ${ }^{201}$, M.S. Kim ${ }^{123}$, T.J. $\operatorname{Kim}^{46}$, V. Kim ${ }^{206,173}$, Y. Kim ${ }^{122}$, R. Kinnunen ${ }^{85}$, M. Kirakosyan ${ }^{166}$, M. Kirsanov ${ }^{160}$, H. Kirschenmann ${ }^{28}$, P. Klabbers ${ }^{235}$, R. Klanner ${ }^{83}$, K. Klapoetke ${ }^{140}$, D. Klein ${ }^{34}$, K. Klein ${ }^{184}$, C. Kleinwort ${ }^{59}$, B. Klima ${ }^{70}$, M. Klute ${ }^{135}$, D. Knowlton ${ }^{155}$, V. Knünz ${ }^{88}$, A. Knutsson ${ }^{6}$, S. Knutzen ${ }^{185}$, W. Ko ${ }^{30}$, S.A. Koay ${ }^{180}$, O. Kodolova ${ }^{202}$, R. Kogler ${ }^{83}$, P. Kokkas ${ }^{110}$, T. Kolberg ${ }^{134}$, G. Kole ${ }^{212}$, J.R. Komaragiri ${ }^{146}$, M. Komm ${ }^{39}$, M. Konecki ${ }^{65}$, D.J. Kong ${ }^{123}$, A. König ${ }^{88}$, J. Konigsberg ${ }^{73}$, 
V. Konoplyanikov ${ }^{116}$, D. Konstantinov ${ }^{208}$, M. Konyushikhin ${ }^{144}$, A. Kornmayer ${ }^{28,66}$, I. Korol ${ }^{59}$, V. Korotkikh ${ }^{202}$, M.J. Kortelainen ${ }^{28}$, A. Korytov ${ }^{73}$, K. Kothekar ${ }^{108}$, D. Kotlinski ${ }^{170}$, K. Kotov ${ }^{163}$, C. Kottachchi Kankanamge Don ${ }^{233}$, K. Kousouris ${ }^{28}$, M. Kovac ${ }^{205}$, N. Kovalchuk ${ }^{83}$, D. Kovalskyi ${ }^{135}$, K. Kovitanggoon ${ }^{48}$, K. Krajczar ${ }^{28}$, M. Krammer 227,88 , N. Krasnikov ${ }^{160}$, I. Krätschmer ${ }^{88}$, I. Kravchenko ${ }^{155}$, L. Kreczko ${ }^{24}$, B. Kreis ${ }^{70}$, T. Kress ${ }^{186}$, P. Kreuzer ${ }^{185}$, D. Krofcheck ${ }^{10}$, M. Krohn ${ }^{50}$, J. Krolikowski ${ }^{65}$, A. Kropivnitskaya ${ }^{118}$, V. Krutelyov ${ }^{216}$, V. Krychkine ${ }^{208}$, A. Kubik ${ }^{157}$, Y. Kubota ${ }^{140}$, Y. Kuessel ${ }^{186}$, A. Kumar ${ }^{57}$, A. Kumar ${ }^{209}$, Arun Kumar ${ }^{153}$, Ashok Kumar ${ }^{57}$, R. Kumar ${ }^{168}$, S. Kumar ${ }^{212}$, V. Kumar ${ }^{19}$, J. Kunkle ${ }^{134}$, R. Kunnawalkam Elayavalli ${ }^{193}$, S. Kunori ${ }^{218}$, A. Künsken ${ }^{186}$, C.M. Kuo ${ }^{144}$, P. Kurt ${ }^{105}$, E. Kuznetsova ${ }^{173}$, S. Kwan ${ }^{70, *}$, E. Kwon ${ }^{211}$, P. Kyberd ${ }^{27}$, A. Kyriakis ${ }^{162}$, C. La Licata ${ }^{103,223}$, F. Lacroix ${ }^{33}$, F. Lagarde ${ }^{131}$, Y.S. Lai ${ }^{135}$, E. Laird ${ }^{26}$, I.B. Laktineh ${ }^{131}$,

K. Lamichhane ${ }^{218}$, P. Lamichhane ${ }^{233}$, S. Lammel ${ }^{70}$, T. Lampén ${ }^{85}$, A. Lanaro ${ }^{235}$, R. Lander ${ }^{30}$, G. Landsberg ${ }^{26}$, R. Lane ${ }^{106}$, A. Lanev ${ }^{116}$, C. Lange ${ }^{224}$, D. Lange ${ }^{128}$, W. Lange ${ }^{59}$, U. Langenegger ${ }^{170}$, K. Lannon ${ }^{158}$, G. Lanza ${ }^{96}$, T. Lapsien $^{83}$, P. Lariccia ${ }^{99,172}$, K. Lassila-Perini ${ }^{85}$, A. Lath ${ }^{193}$, J. Lauwers ${ }^{6}$, P. Lawson ${ }^{22}$, A.-C. Le Bihan ${ }^{178}$, P. Lecoq ${ }^{28}$, A. Ledovskoy ${ }^{229}$, B. Lee ${ }^{122}$, J. Lee ${ }^{211}$, J.S.H. Lee ${ }^{201}$, K. Lee ${ }^{122}$, K.S. Lee ${ }^{122}$, S. Lee ${ }^{123}$, S. Lee ${ }^{122}$, S.W. Lee ${ }^{218}$, Y.-J. Lee ${ }^{135}$, D. Leggat ${ }^{27}$, S. Lehti ${ }^{85}$, D. Lelas ${ }^{204}$, V. Lemaitre ${ }^{39}$, T. Lenz ${ }^{83}$, P. Lenzi ${ }^{93,71}$, T. Lenzi ${ }^{129}$, A. Léonard ${ }^{129}$, J. Leonard ${ }^{59}$, N. Leonardo ${ }^{126}$, A. Leonidov ${ }^{151,166}$, Z. Lesko ${ }^{140}$, D. Leslie ${ }^{27}$, M. Lethuillier ${ }^{131}$, J. Letts ${ }^{34}$, P. Levchenko ${ }^{173}$, L. Levchuk ${ }^{152}$, A. Levin ${ }^{135}$, A. Levine ${ }^{235}, \mathrm{H}_{\text {. Li }}{ }^{229}$, Q. Li $^{207}$, W. Li ${ }^{188}$, T. Libeiro ${ }^{218}$, F. Ligabue ${ }^{100,198}$, D. Liko ${ }^{88}$, C. Lin ${ }^{229}$, W. Lin ${ }^{144}$, J. Linacre ${ }^{70}$, D. Lincoln ${ }^{70}$, T. Lindén ${ }^{85}$, T.Y. Ling ${ }^{163}$, J. Lingemann ${ }^{186}$, S. Linn ${ }^{75}$, M. Lipinski ${ }^{184}$, K. Lipka ${ }^{59}$, R. Lipton ${ }^{70}$, S. Lisniak ${ }^{124}$, L. Lista ${ }^{96}$, L. Litov ${ }^{203}$, B. Liu ${ }^{163}$, H. Liu ${ }^{15}$, S. Liu ${ }^{207}$, T. Liu $^{70}$, Y.F. Liu ${ }^{153}$, L. Lloret Iglesias ${ }^{126}$, M. Lo Vetere ${ }^{94,79}$, A. Lobanov ${ }^{59}$, P. Lobelle Pardo ${ }^{66}$, E. Locci ${ }^{55}$, W. Lohmann ${ }^{23,59}$, I. Lokhtin ${ }^{202}$, T. Lomtadze ${ }^{100}$, K. Long ${ }^{235}$, O.R. Long ${ }^{33}$, E. Longo ${ }^{101,190}$, R. Lopes De Sá ${ }^{70}$, A. Lopez Virto ${ }^{72}$, R. Lopez-Fernandez ${ }^{43}$, D. Loukas ${ }^{162}$, N. Loukas ${ }^{110}$, C. Lourenço ${ }^{28}$, R. Loveless ${ }^{235}$, J.F. Low ${ }^{73}$, S. Lowette ${ }^{231}$, R.-S. Lu ${ }^{153}$, Y. Lu ${ }^{134}$, Y.J. Lu ${ }^{144}$, C. Lucas ${ }^{24}$, R. Lucas ${ }^{194,106}$, M.T. Lucchini ${ }^{28}$, P.D. Luckey ${ }^{135}$, J. Luetic ${ }^{191}$, P. Lujan ${ }^{180}$, W. Luo ${ }^{163}$, W. Lustermann ${ }^{169}$, A. Luthra ${ }^{33}$, P. Luukka ${ }^{85}$, S. Luyckx ${ }^{6}$, 
N. Lychkovskaya ${ }^{219}$, J. Lykken ${ }^{70}$, L. Lyons ${ }^{106}$, P. Ma $^{73}$, M. Machet ${ }^{55}$, I. Macneill ${ }^{34}$, T. Mäenpää ${ }^{85}$, T. Maerschalk ${ }^{129}, \mathrm{~K}$. Maeshima ${ }^{70}$, G. Maggi ${ }^{90,179}$, M. Maggi ${ }^{90}$, N. Magini ${ }^{28}$, A.-M. Magnan ${ }^{106}$, A. Magnani ${ }^{98}$, C. Maguire ${ }^{226}$, B. Mahakud ${ }^{212}$, A. Mahrous ${ }^{86,1}$, B. Maier ${ }^{66}$, M. Maity ${ }^{230,212}$, N. Majumdar ${ }^{195}$, D. Majumder ${ }^{119}$, G. Majumder ${ }^{212}$, M. Makouski ${ }^{120}$, A. Makovec ${ }^{56}$, P. Maksimovic ${ }^{115}$, P. Mal ${ }^{150}$, A. Malakhov ${ }^{116}$, M. Malberti ${ }^{33}$, H. Malbouisson ${ }^{62}$, J. Malcles ${ }^{55}$, M. Malek ${ }^{119}$, L. Malgeri ${ }^{28}$, S. Malhotra ${ }^{57}$, S. Malik ${ }^{181}$, S. Malik ${ }^{106}$, S. Malvezzi ${ }^{95}$, K. Mandal ${ }^{150}$, B. Mangano ${ }^{169}$, R. Mankel ${ }^{59}$, M. Mannelli ${ }^{28}$, J. Mans ${ }^{140}$, N. Manthos ${ }^{110}$, G. Mantovani ${ }^{99,172}$, R.A. Manzoni ${ }^{28,95,138}$, Y. Mao ${ }^{207}$, Y. Mao ${ }^{226}$, Z. Mao ${ }^{26}$, Y. Maravin ${ }^{120}$, S. Marcellini ${ }^{91}$, I. Marchesini ${ }^{83}$, J. Marco ${ }^{72}$, R. Marco ${ }^{72}$, D. Marconi ${ }^{83}$, I. Marfin ${ }^{23,59}$, F. Margaroli ${ }^{101,190}$, M. Margoni ${ }^{97,167}$, N. Marinelli ${ }^{158}$, A.C. Marini ${ }^{135}$, A. Marinov ${ }^{129}$, M. Marionneau ${ }^{169}$, C. Mariotti ${ }^{102}$, P. Markowitz ${ }^{75}$, D. Marlow ${ }^{180}$, G. Maron ${ }^{125,97}$, M. Marone ${ }^{103,223}$, J.M. Marraffino ${ }^{70}$, A. Martelli ${ }^{28}$, C. Martin ${ }^{115}$, C. Martinez Rivero ${ }^{72}$, G. Martinez ${ }^{75}$, P. Martinez Ruiz del Arbol ${ }^{169}$,

V.I. Martinez Outschoorn ${ }^{70}$, L. Martini ${ }^{100,176}$, S. Maruyama ${ }^{70}$, B. Marzocchi ${ }^{28,95,138}$, M. Masciovecchio ${ }^{169}$, S. Maselli ${ }^{102}$, G. Masetti ${ }^{91}$, L. Masetti ${ }^{28}$, D. Mason ${ }^{70}$, A. Massironi ${ }^{156}$, L. Mastrolorenzo ${ }^{124}$, K. Matchev ${ }^{73}$, F. Matorras ${ }^{72}$, D. Matos Figueiredo ${ }^{62}$, T. Matsushita ${ }^{88}$, V. Matveev ${ }^{160,151,116}$, G. Mavromanolakis ${ }^{54}$, K. Mazumdar ${ }^{212}$, P. McBride ${ }^{70}$, J. Mccartin ${ }^{81}$, N. Mccoll ${ }^{35}$, C. Mcginn ${ }^{135}$, M.A.B. Md Ali ${ }^{109,146}$, T. Medvedeva ${ }^{180}$, A. Mehta ${ }^{168}, \mathrm{H} . \mathrm{Mei}^{73}$, F. Meier ${ }^{155}$, F. Meijers ${ }^{28}$, D. Meister ${ }^{169}$, A. Melo ${ }^{226}$, I.-A. Melzer-Pellmann ${ }^{59}$, D. Menasce ${ }^{95}$, A.T. Meneguzzo ${ }^{97,167}$, F. Meng ${ }^{158}$, Z. Meng ${ }^{24}$, M. Menichelli ${ }^{99}$, S. Meola ${ }^{28,96,77}$, P.G. Mercadante ${ }^{69}$, P. Meridiani ${ }^{101}$, P. Merkel ${ }^{70}$, J.A. Merlin ${ }^{28,178}$, J.-P. Merlo ${ }^{111}$, H. Mermerkaya ${ }^{61,111}$, M. Merola ${ }^{96}$, M. Merschmeyer ${ }^{185}$, S. Mersi ${ }^{28}$, A. Mertens ${ }^{39}$, E. Meschi ${ }^{28}$, M. Meschini ${ }^{93}$, A. Messineo ${ }^{100,176}$, A. Mestvirishvili ${ }^{111}$, G. Mesyats ${ }^{166}$, A. Meyer ${ }^{185}$, A.B. Meyer ${ }^{59}$, M. Meyer ${ }^{83}$, M. Miñano Moya ${ }^{153}$, S. Micanovic ${ }^{191}$, F. Micheli ${ }^{169}$, M. Michelotto ${ }^{97}$, B. Michlin ${ }^{188}$, E. Migliore ${ }^{102,220}$, A.C. Mignerey ${ }^{134}$, I. Mikulec ${ }^{88}$, H. Mildner ${ }^{66}$, P. Milenovic ${ }^{16,73}$, D.H. Miller ${ }^{183}$, P. Millet ${ }^{185}$, J. Milosevic ${ }^{16}$, P. Miné ${ }^{124}$, G. Miniello ${ }^{90,13}$, L. Mirabito ${ }^{131}$, N. Mirman ${ }^{52}$, C. Mironov ${ }^{135}, \mathrm{~K}$. Mishra ${ }^{70}$, M. Misiura ${ }^{65}$, M. Missiroli ${ }^{11}$, S. Mitra ${ }^{212}$, G. Mitselmakher ${ }^{73}$, G. Mittag ${ }^{59}$, 


\section{Mittal ${ }^{168}$, J. Mnich ${ }^{59}$, A. Modak ${ }^{195}$, A. Moeller ${ }^{111}$, F. Mohamad Idris ${ }^{132,146}$, A. Mohammadi ${ }^{120}$,}

M. Mohammadi Najafabadi ${ }^{187}$, A.K. Mohanty ${ }^{28,19}$, G.B. Mohanty ${ }^{212}$,

A. Mohapatra ${ }^{235}$, P. Moisenz ${ }^{116}$, J. Molnar ${ }^{159}$, V. Monaco ${ }^{102,220}$, K. Mondal ${ }^{195}$, M.R. Monge ${ }^{94,79}$, J. Monroy ${ }^{155}$, P. Montagna ${ }^{98,171}$, A. Montanari ${ }^{91}$, F. Montecassiano ${ }^{97}$, E. Monteil ${ }^{102,220}$, C.S. Moon ${ }^{40,63}$, M. Mooney ${ }^{180}$, F. Moortgat ${ }^{28}$, C. Mora Herrera ${ }^{42}$, A. Moraes ${ }^{42}$, D. Moran ${ }^{11}$, L. Moreels ${ }^{231}$, A. Morelos Pineda ${ }^{12}$, L. Moroni ${ }^{95}$, S. Morovic ${ }^{28}$, D.M. Morse ${ }^{156}$, V. Mossolov ${ }^{147}$, A. Mott ${ }^{31}$, J. Mousa ${ }^{54}$, M.U. Mozer ${ }^{66}$, S. Mrenna ${ }^{70}$, N. Mucia ${ }^{157}$, C. Mueller ${ }^{158}$, R. Mueller ${ }^{216}$, S. Mukherjee ${ }^{195}$, S. Mukhopadhyay ${ }^{195}$, M. Mulders ${ }^{28}$, M. Mulhearn ${ }^{30}$, T. Mulholland ${ }^{50}$, T. Müller ${ }^{66}$, Th. Müller ${ }^{66}$, S.D. Mullin ${ }^{35}$, L. Mundim ${ }^{62}$, M. Murray ${ }^{119}$, M. Murumaa ${ }^{149}$, V. Murzin ${ }^{173}$, P. Musella ${ }^{169}$, M. Musich ${ }^{39}$, Y. Musienko ${ }^{160,158}$, A. Mussgiller ${ }^{59}$, S. My ${ }^{90,179}$, I. Myagkov ${ }^{202}$, J. Nachtman ${ }^{111}$, S. Nahn ${ }^{70}$, M. Naimuddin ${ }^{57}$, S.K. Nam ${ }^{118}$, M. Narain ${ }^{26}$, I.N. Naranjo ${ }^{124}$, S. Narayanan ${ }^{135}$, M. Naseri ${ }^{187}$, D. Nash ${ }^{156}$, J. Nash ${ }^{106}$, K. Nash ${ }^{193}$, U. Nauenberg ${ }^{50}$, S. Naumann-Emme ${ }^{59}$, F.L. Navarria ${ }^{91,21}$, E. Navarro De Martino ${ }^{44}$, K. Nawrocki ${ }^{145}$, A. Nayak ${ }^{59}$, A. Nehrkorn ${ }^{186}$, M.V. Nemallapudi ${ }^{28}$, F. Nessi-Tedaldi ${ }^{169}$, C. Neu ${ }^{229}$, H. Neugebauer ${ }^{28}$, N. Neumeister ${ }^{183}$, D.M. Newbold ${ }^{194,24}$, H.B. Newman ${ }^{31}$, C. Newman-Holmes ${ }^{70}$, J. Ngadiuba ${ }^{224}$, F. Nguyen ${ }^{126}$, M. Nguyen ${ }^{124},{\mathrm{H} . ~ \mathrm{Ni}^{226} \text {, C. Nicolaou }}^{54}$, G. Nicolas Kaufman ${ }^{52}$, A. Nikitenko ${ }^{219,106}$, N. Nishu ${ }^{57}$, X. Niu ${ }^{135}$, H. Nogima ${ }^{62}$, D. Noonan ${ }^{74}$, M. Northup ${ }^{188}$, S. Nourbakhsh ${ }^{140}$, S.F. Novaes ${ }^{63}$, A. Nowack ${ }^{186}$, D. Nowatschin ${ }^{83}$, E. Ntomari ${ }^{59}$, I.M. Nugent ${ }^{186}$, C. Nuttens ${ }^{39}$, S. Nuzzo ${ }^{90,13}$, C. O’Brien ${ }^{105}$, V. O’Dell ${ }^{70}$, M.M. Obertino ${ }^{102,220}$, S. Obraztsov ${ }^{202}$, A.A. Ocampo Rios ${ }^{81}$, C. Ochando ${ }^{124}$, N. Odell ${ }^{157}$, H. Ogul ${ }^{111}$, Y.D. Oh ${ }^{123}$, I. Ojalvo ${ }^{235}$, E. Olaiya ${ }^{194}$, A. Olbrechts ${ }^{231}$, S. Oliveros ${ }^{141}$, D. Olivito ${ }^{34}$, M. Olmedo Negrete ${ }^{33}$, M. Olschewski ${ }^{185}$, J. Olsen ${ }^{180}$, M. Olszewski ${ }^{65}$, Y. Onel ${ }^{111}$, G. Onengut ${ }^{29,53}$, V. Oreshkin ${ }^{173}$, S. Orfanelli ${ }^{154,28}$, G. Organtini ${ }^{101,190}$, T. Orimoto ${ }^{156}$, L. Orsini ${ }^{28}$, G. Ortona ${ }^{124}$, M. Osherson ${ }^{115}$, I. Osipenkov ${ }^{216}$, A. Ostapchuk ${ }^{184}$, J. Ott $^{83}$, K. Ozdemir ${ }^{175,53}$, F. Ozok ${ }^{139,111}$, S. Ozturk ${ }^{78,53}$, L. Pacher ${ }^{102,220}$, K. Padeken ${ }^{185}$, S. Padhi ${ }^{34}$, B.P. Padley ${ }^{188}$, P. Paganini ${ }^{124}$, M. Paganoni ${ }^{95,138}$, Y. Pakhotin ${ }^{216}$, S. Paktinat Mehdiabadi ${ }^{187}$, E. Palencia Cortezon ${ }^{164}$, V. Palichik ${ }^{116}$, F. Palla ${ }^{100}$, C. Palmer ${ }^{180}$, 
A. Panagiotou ${ }^{9}$, F. Pandolfi ${ }^{169}$, L.M. Pant ${ }^{19}$, F. Pantaleo ${ }^{28,83}$,

S. Panwalkar ${ }^{193}$, S. Paoletti ${ }^{93}$, P. Paolucci ${ }^{28,96}$, P. Papacz ${ }^{185}$,

I. Papadopoulos ${ }^{110}$, L. Pape ${ }^{28}$, E. Paradas ${ }^{110}$, R. Paramatti ${ }^{101}$,

S. Paramesvaran ${ }^{24}$, N. Parashar ${ }^{182}$, B. Parida ${ }^{212}$, I.C. Park ${ }^{201}$, M. Park ${ }^{193}$,

S.K. Park ${ }^{122}$, A. Pashenkov ${ }^{160}$, M. Passaseo ${ }^{97}$, N. Pastika ${ }^{15}$,

N. Pastrone ${ }^{102}$, J. Pata ${ }^{169}$, R. Patel ${ }^{216}$, J.R. Patterson ${ }^{52}$, M. Paulini ${ }^{37}$,

C. Paus ${ }^{135}$, F. Pauss ${ }^{169}$, B. Pavlov ${ }^{203}$, J. Pazzini ${ }^{28,97,167}$, I. Pedraza ${ }^{17}$,

D. Pedrini ${ }^{95}$, K. Pedro ${ }^{70}$, M. Pegoraro ${ }^{97}$, T. Peiffer ${ }^{83}$, J. Pekkanen ${ }^{58}$,

J. Pela ${ }^{106}$, D. Pellett ${ }^{30}$, M. Pelliccioni ${ }^{102}$, T. Peltola ${ }^{85}$, C. Pena ${ }^{31}$,

A. Penzo ${ }^{111}$, A.L. Pequegnot ${ }^{131}$, V. Perelygin ${ }^{116}$, E. Perez ${ }^{28}$,

A. Pérez-Calero Yzquierdo ${ }^{44}$, A. Perieanu ${ }^{83}$, A. Perloff ${ }^{216}$, L. Perniè ${ }^{129}$,

H. Perrey ${ }^{59}$, S. Perries ${ }^{131}$, L. Perrini ${ }^{39}$, A. Perrotta ${ }^{91}$, L. Perrozzi ${ }^{169}$, T. Perry ${ }^{235}$, M. Peruzzi ${ }^{28}$, M. Pesaresi ${ }^{106}$, P. Petkov ${ }^{203}$, E. Petrakou ${ }^{153}$, K. Petridis ${ }^{106}$, A. Petrilli ${ }^{28}$, G. Petrillo ${ }^{189}$, V. Petrov ${ }^{208}$, G. Petrucciani ${ }^{28}$, S. Petrushanko ${ }^{202}$, D. Petyt ${ }^{194}$, A. Pfeiffer ${ }^{28}$, D. Piccolo ${ }^{89}$, J. Piedra Gomez ${ }^{72}$, M. Pieri ${ }^{34}$, M. Pierini ${ }^{31}$, G.A. Pierro ${ }^{235}$, N. Pietsch ${ }^{83}$, P. Pigard ${ }^{124}$, J. Pilot ${ }^{30}$, A. Pin ${ }^{39}$, D. Pinna ${ }^{224}$, G.L. Pinna Angioni ${ }^{102,220}$, K. Piotrzkowski ${ }^{39}$, D. Piparo ${ }^{28}$, S. Piperov ${ }^{26}$, P. Piroué ${ }^{180}$, C. Pistone ${ }^{186}$, D. Pitzl ${ }^{59}$, R. Placakyte ${ }^{59}$, M. Plagge ${ }^{66}$, M. Planer ${ }^{158}$, R. Plestina ${ }^{124,87}$, J. Poehlsen ${ }^{83}$, M.E. Pol ${ }^{42}$, G. Polese ${ }^{235}$, A. Poll ${ }^{24}$, B. Pollack ${ }^{157}$, A. Pompili ${ }^{90,13}$, T. Pook ${ }^{185}$, O. Pooth ${ }^{186}$, A. Popov ${ }^{202,39}$, V. Popov ${ }^{219}$, R. Potenza ${ }^{92,38}$, D. Poyraz ${ }^{81}$, A. Pozdnyakov ${ }^{157}$, I. Pozdnyakov ${ }^{219}$, N. Pozzobon ${ }^{97,167}$, W.L. Prado Da Silva ${ }^{62}$, F. Preiato ${ }^{101,190}$, M. Preuten ${ }^{184}$,

F. Primavera ${ }^{28,89}$, O. Prokofyev ${ }^{70}$, H. Prosper ${ }^{76}$, A. Psallidas ${ }^{162}$,

F. Ptochos ${ }^{54}$, J. Puerta Pelayo ${ }^{44}$, G. Pugliese ${ }^{90,179}$, D. Puigh ${ }^{163}$, I. Puljak ${ }^{204}$, Q. Python ${ }^{231}$, S.J. Qian ${ }^{207}$, G. Quast ${ }^{66}$, L. Quertenmont ${ }^{39}$, A. Quintario Olmeda ${ }^{44}$, M. Quittnat ${ }^{169}$, B.R. Yates ${ }^{33}$, D. Rabady ${ }^{28,88}$, K. Rabbertz ${ }^{66}$, A. Racz ${ }^{28}$, B.C. Radburn-Smith ${ }^{183}$, A. Radi ${ }^{25,3,1}$, R. Radogna ${ }^{90,13}$, M. Radziej ${ }^{185}$, S. Ragazzi ${ }^{95,138}$, S. Rahatlou ${ }^{101,190}$, B. Rahbaran ${ }^{88}$, P. Raics ${ }^{56}$, M. Raidal ${ }^{149}$, G. Rakness ${ }^{70}$, D. Ralph ${ }^{135}$, J. Rander ${ }^{55}$, A. Randle-conde ${ }^{129}$, A. Ranieri ${ }^{90}$, K. Ranjan ${ }^{57}$, D. Rank ${ }^{73}$, D. Rankin ${ }^{22}$, S. Rappoccio ${ }^{209}$, A. Raspereza ${ }^{59}$, D. Rathjens ${ }^{83}$, F. Ratnikov ${ }^{155}$, S.P. Ratti ${ }^{98,171}$, F. Raupach ${ }^{184}$, F. Ravera ${ }^{102,220}$, D.M. Raymond ${ }^{106}$, P.A. Razis ${ }^{54}$, V. Re ${ }^{98}$, F. Rebassoo ${ }^{128}$, P. Rebello Teles ${ }^{42}$, N. Redaelli ${ }^{95}$, R. Redjimi ${ }^{188}$, I. Redondo ${ }^{44}$, S. Regnard ${ }^{124}$, I.D. Reid ${ }^{27}$, A. Reinsvold ${ }^{158}$, T. Reis ${ }^{28}$, H. Reithler ${ }^{185}$, V. Rekovic ${ }^{16}$, D. Renker ${ }^{170}$, F. Rezaei Hosseinabadi ${ }^{187}$, 
P.M. Ribeiro Cipriano ${ }^{204}$, C. Riccardi ${ }^{98,171}$, F. Ricci-Tam ${ }^{30}$, A. Richards ${ }^{106}$, C. Richardson ${ }^{22}$, J. Richman ${ }^{35}$, M. Rieger ${ }^{185}$, G. Riley ${ }^{215}$, A. Rinkevicius ${ }^{52}$, A. Rizzi ${ }^{100,176}$, J. Roberts ${ }^{188}$, P. Robmann ${ }^{224}$, E. Robutti ${ }^{94}$, S. Röcker ${ }^{66}$, M. Rodenburg ${ }^{163}$, M. Rodozov ${ }^{161}$, T. Rodrigo ${ }^{72}$, J. Rodrigues Antunes ${ }^{126}$, J.L. Rodriguez ${ }^{75}$, A.Y. Rodríguez-Marrero ${ }^{72}$, Y. Roh ${ }^{122}$, T. Rohe ${ }^{170}$, J. Rohlf ${ }^{22}$, H. Rohringer ${ }^{88}$, B. Roland ${ }^{59}$, C. Roland ${ }^{135}$, G. Roland ${ }^{135}$, G. Rolandi ${ }^{199,28}$, K. Romanowska-Rybinska ${ }^{145}$, F. Romeo ${ }^{87}$, A. Romero ${ }^{102,220}$, D. Romero Abad ${ }^{63,69}$, L. Romero ${ }^{44}$, P. Ronchese ${ }^{97,167}$, F.J. Ronga ${ }^{224}$, B. Roozbahani ${ }^{209}$, J. Rorie ${ }^{188}$, F. Roscher ${ }^{66}$, A. Rose ${ }^{216}$, A. Rose ${ }^{106}$, K. Rose $^{215}$, J. Roskes ${ }^{115}$, A. Rosowsky ${ }^{55}$, A.M. Rossi ${ }^{91,21}$, R. Rossin ${ }^{73}$, M. Rossini ${ }^{169}$, C. Rovelli ${ }^{101}$, T. Rovelli ${ }^{91,21}$, M. Rovere ${ }^{28}$, A. Roy ${ }^{195}$, D. Roy ${ }^{195}$, S. Roy Chowdhury ${ }^{195}$, T. Roy ${ }^{74}$, M. Ruan ${ }^{28}$,

R. Ruchti ${ }^{158}$, N. Ruckstuhl ${ }^{140}$, T. Ruggles ${ }^{235}$, J.C. Ruiz Vargas, J.D. Ruiz Alvarez ${ }^{131}$, A. Ruiz-Jimeno ${ }^{72}$, P. Rumerio ${ }^{4}$, R. Rusack ${ }^{140}$, S.V. Rusakov ${ }^{166}$, M. Ruspa ${ }^{102,174}$, J. Russ ${ }^{37}$, D. Ryckbosch ${ }^{81}$, A. Ryd ${ }^{52}$, H. Rykaczewski ${ }^{54}$, G. Ryu ${ }^{201}$, M.S. Ryu ${ }^{201}$, R. Ryutin ${ }^{208}$, Sandra S. Padula ${ }^{63}$, D. Sabes ${ }^{131}$, R. Sacchi ${ }^{102,220}$, A. Sady ${ }^{115}$, B. Safarzadeh ${ }^{177,187}$, A. Safonov ${ }^{216}$, G. Safronov ${ }^{219}$, S. Sagir ${ }^{26}$, A. Saha ${ }^{99}$, M.Ö. Sahin ${ }^{59}$, D.K. Sahoo ${ }^{150}$, N. Sahoo ${ }^{150}$, L.K. Saini ${ }^{120}$, H. Saka ${ }^{180}$, A. Sakharov ${ }^{123}$, H. Sakulin ${ }^{28}$, T. Sakuma ${ }^{24}$, H.A. Salazar Ibarguen ${ }^{17}$, D. Salerno ${ }^{224}$, R. Salerno ${ }^{124}$, J. Salfeld-Nebgen ${ }^{135}$, D. Saltzberg ${ }^{32}$, S. Salur ${ }^{193}$, S. Salva ${ }^{81}$, P. Salvini ${ }^{98}$, J.C. Sanabria ${ }^{130}$, A. Sanchez-Hernandez ${ }^{43}$, C. Sander ${ }^{83}$, S. Sanders ${ }^{119}$, I.D. Sandoval Gonzalez ${ }^{105}$, M. Sani ${ }^{34}$, F. Santanastasio ${ }^{101,190,}$ J. Santaolalla ${ }^{44}$, A. Santocchia ${ }^{99,172}$, A. Santoro ${ }^{62}$, N. Saoulidou ${ }^{9}$, T. Sarangi ${ }^{235}$, U. Sarica ${ }^{115}$, S. Sarkar ${ }^{195}$, T. Sarkar ${ }^{230,212}$, J.B. Sauvan ${ }^{124}$, A. Savin ${ }^{235}$, A. Savoy-Navarro ${ }^{183,100}$, V. Savrin ${ }^{202}$, P. Saxena ${ }^{59}$, S. Schael ${ }^{184}$, C. Schäfer ${ }^{28}$, C. Scharf ${ }^{83}$, H. Schettler ${ }^{83}$, F. Scheuch ${ }^{185}$, J. Schieck ${ }^{227,88}$, A. Schizzi ${ }^{103,223}$, P. Schleper ${ }^{83}$, E. Schlieckau ${ }^{83}$, A. Schmidt ${ }^{83}$, M. Schmitt ${ }^{157}$, S. Schnetzer ${ }^{193}$, T. Schoerner-Sadenius ${ }^{59}$, R. Schöfbeck ${ }^{88}$, M. Schröder ${ }^{59}$, J.F. Schulte ${ }^{184}$, J. Schwandt ${ }^{83}$, C. Schwick ${ }^{28}$, C. Sciacca ${ }^{96,143}$, L. Scodellaro ${ }^{72}$, C. Seez ${ }^{106}$, M. Seidel ${ }^{28}$, S. Seif El Nasr-storey ${ }^{24}$, C. Seitz ${ }^{59}$, J. Seixas ${ }^{126}$, G. Selvaggi ${ }^{90,13}$, M. Selvaggi ${ }^{39}$, S. Sen ${ }^{82,113}$, S. Senkin ${ }^{24}$, A.T. Serban ${ }^{100}$, T. Seva ${ }^{129}$, E. Sexton-Kennedy ${ }^{70}$, L. Sgandurra ${ }^{131}$, G. Sguazzoni ${ }^{93}$, S.M. Shaheen ${ }^{87}$, S. Shalhout ${ }^{30}$, M. Sharan ${ }^{195}$, A. Sharma ${ }^{235}$, A. Sharma ${ }^{28}$, R. Sharma ${ }^{57}$, 
S. Sharma ${ }^{108}$, V. Sharma ${ }^{57}$, V. Sharma ${ }^{34}$, L. Shchutska ${ }^{73}$, D. Sheffield ${ }^{193}$, P. Sheldon ${ }^{226}$, C.H. Shepherd-Themistocleous ${ }^{194}$, X. Shi ${ }^{183}$, Y.H. Shin ${ }^{134}$, I. Shipsey ${ }^{183}$, S. Shmatov ${ }^{116}$, M. Shoaib ${ }^{148}$, A. Shrinivas ${ }^{33}$, P. Shukla ${ }^{19}$, S. Shulha ${ }^{116}$, N. Shumeiko ${ }^{147}$, J.E. Siado ${ }^{155}$, G. Sieber ${ }^{66}$, M. Sigamani ${ }^{81}$, F. Sikler ${ }^{234}$, C. Silkworth ${ }^{105}$, P. Silva ${ }^{28}$, D. Silvers ${ }^{183}$, L. Silvestris ${ }^{28,90}$, M. Simon ${ }^{28}$, S. Simon ${ }^{34}$, F. Simonetto ${ }^{97,167}$, H.J. Simonis ${ }^{66}$, G. Singh ${ }^{48}$, J.B. Singh ${ }^{168}$, T. Sinthuprasith ${ }^{229}$, Y. Sirois ${ }^{124}$, G.P. Siroli ${ }^{91,21}$, A.M. Sirunyan ${ }^{236}$, N. Skatchkov ${ }^{116}$, N. Skhirtladze ${ }^{120}$, L. Skinnari ${ }^{52}$, K. Skovpen ${ }^{178}$, A. Skuja ${ }^{134}$, I. Smirnov ${ }^{173}$, V. Smirnov ${ }^{116}$, D. Smith ${ }^{24}$, G. Smith ${ }^{158}$, J. Smith ${ }^{30}$, N. Smith ${ }^{235}$, V.J. Smith ${ }^{24}$, W.H. Smith ${ }^{235}$, A. Snigirev ${ }^{202}$, B. Snook ${ }^{226}$, G.R. Snow ${ }^{155}$, M. Snowball ${ }^{73}$, C. Snyder ${ }^{111}$, M.S. Soares ${ }^{44}$, A. Sobol ${ }^{208}$, L. Soffi ${ }^{52}$, A. Soha ${ }^{70}$, V. Sola ${ }^{83}$, A. Solano ${ }^{102,220}$, S. Somalwar ${ }^{193}$, D.C. Son ${ }^{123}$, S. Song ${ }^{47}$, L. Sonnenschein ${ }^{185}$, V. Sordini ${ }^{131}$, P. Sorokin ${ }^{152}$, P. Spagnolo ${ }^{100}$, W.J. Spalding ${ }^{70}$, S. Spanier ${ }^{215}$, S. Spannagel ${ }^{59}$, D. Sperka ${ }^{73}$, P. Sphicas ${ }^{9,28}$, L. Spiegel ${ }^{70}$, A. Spiezia ${ }^{87}$, A. Spiridonov ${ }^{219}$, M. Spiropulu ${ }^{31}$, M. Squires ${ }^{30}$, N. Srimanobhas ${ }^{48}$, J. St. John ${ }^{22}$, H. Stadie ${ }^{83}$, A. Stahl ${ }^{186}$, A. Staiano ${ }^{102}$, A. Starodumov ${ }^{219,169}$, J. Steggemann ${ }^{28}$, G. Steinbrück ${ }^{83}$, K. Stenson ${ }^{50}$, G.S.F. Stephans ${ }^{135}$, D. Stickland ${ }^{180}$, B. Stieger ${ }^{28}$, F.M. Stober ${ }^{66}$, D. Stolp ${ }^{30}$, R. Stone ${ }^{193}$, M. Stoye ${ }^{28}$, S. Stoykova ${ }^{161}$, S. Stoynev ${ }^{157}$, J. Strauss ${ }^{88}$, T. Strebler ${ }^{124}$, R. Stringer ${ }^{119}$, N. Strobbe ${ }^{70}$, J. Strologas ${ }^{110}$, D. Strom ${ }^{231}$, D. Stuart ${ }^{35}$, J. Stupak ${ }^{182}$, J. Sturdy ${ }^{233}$, I. Suarez ${ }^{35}$, J. Suarez Gonzalez ${ }^{147}$, L. Sudic ${ }^{191}$, L. Sulak ${ }^{22}$, V. Sulimov ${ }^{173}$, G. Sultanov ${ }^{161}$, K. Sumorok ${ }^{135}$, J. Sun ${ }^{183}$, M. Sun ${ }^{37}$, W. Sun ${ }^{52}$, X. Sun ${ }^{229}$, K. Sung ${ }^{157}$, N. Sur ${ }^{212}$, B. Sutar ${ }^{212}$, N. Suwonjandee ${ }^{48}$, A. Svyatkovskiy ${ }^{183}$, S.K. Swain ${ }^{150}$, M. Swartz ${ }^{115}$, R. Syarif ${ }^{26}$, P. Symonds ${ }^{27}$, Z. Szillasi ${ }^{28,159}$, M. Szleper ${ }^{145}$, A. Sznajder ${ }^{62}$, T. Tabarelli de Fatis ${ }^{95,138}$, M. Tadel ${ }^{34}$, M. Takahashi ${ }^{169}$, Y. Takahashi ${ }^{28}$, E. Takasugi ${ }^{32}$, B. Tali ${ }^{2,53}$, J. Talvitie ${ }^{127}$, N. Tambe ${ }^{140}$, P. $\operatorname{Tan}^{189}$, S.M. Tan ${ }^{52}$, J. Tao ${ }^{87}$, A. Tapper ${ }^{106}$, S. Taroni ${ }^{158}$, A. Tatarinov ${ }^{216}$, S. Tavernier ${ }^{231}$, V.R. Tavolaro ${ }^{169}$, D. Taylor ${ }^{235}$, L. Taylor ${ }^{70}$, R. Teixeira De Lima ${ }^{156}$, R. Tenchini ${ }^{100}$, W.D. Teo ${ }^{52}$, L. Teodorescu ${ }^{27}$, N. Terentyev ${ }^{73}$, D. Teyssier ${ }^{185}$, A. Thea ${ }^{194}, \mathrm{~K}$. Theofilatos ${ }^{169}$, H. Tholen ${ }^{83}$, J. Thom ${ }^{52}$, L. Thomas ${ }^{73}$, S. Thomas ${ }^{193}$, P. Thomassen ${ }^{193}$, J. Thompson ${ }^{52}$, S. Thüer ${ }^{185}$, F. Thyssen ${ }^{14,77,96,143}$, A. Tiko ${ }^{149}$, E. Tiras ${ }^{111}$, M. Titov ${ }^{55}$, S. Tkaczyk ${ }^{70}$, D. Tlisov ${ }^{160}$, S. Toda ${ }^{120}$, O. Toldaiev ${ }^{126}$, I.R. Tomalin ${ }^{194}$, E.J. Tonelli Manganote ${ }^{64,62}$, G. Tonelli ${ }^{100,176}$, 
M.B. Tonjes ${ }^{134}$, S.C. Tonwar ${ }^{134}$, H. Topakli ${ }^{78,53}$, A. Topkar ${ }^{19}$, I. Topsis-Giotis ${ }^{162}$, E. Torassa ${ }^{97}$, T. Toriashvili ${ }^{213,80}$, A. Toropin ${ }^{160}$, M. Tosi ${ }^{97,167}$, N. Tosi ${ }^{28,91,21}$, S. Tosi ${ }^{94,79}$, L. Tourtchanovitch ${ }^{208}$, P. Traczyk $^{28,101,190}$, N.V. Tran ${ }^{70}$, H. Trauger ${ }^{105}$, R. Travaglini ${ }^{91,21}$, W. Treberer-Treberspurg ${ }^{88}$, D. Treille ${ }^{28}$, N. Trevisani ${ }^{72}$, A. Tricomi ${ }^{92,38}$, A. Triossi ${ }^{28}$, M. Tripathi ${ }^{30}$, K.D. Trippkewitz ${ }^{59}$, D. Trocino ${ }^{156}$, Z.L. Trocsanyi ${ }^{56}$, D. Troendle ${ }^{83}$, A. Tropiano ${ }^{93,71}$, S. Troshin ${ }^{208}$, M. Trovato ${ }^{157}$, J.f. Tsai ${ }^{153}$, Z. Tsamalaidze ${ }^{116,213}$, A. Tsirou ${ }^{28}$, Z. Tu ${ }^{188}$, J. Tucker ${ }^{52}$, C. Tully ${ }^{180}$, A. Tumasyan ${ }^{236}$, S. Tuo ${ }^{226}$, E. Tuominen ${ }^{85}$, J. Tuominiemi ${ }^{85}$, E. Tuovinen ${ }^{85}$, J. Turkewitz ${ }^{140}$, M. Turner ${ }^{27}$, P. Turner ${ }^{105}$, T. Tuuva ${ }^{127}$, C. Tuve ${ }^{92,38}$, M. Tytgat ${ }^{81}$, N. Tyurin ${ }^{208}$, Y.M. Tzeng ${ }^{153}$, E. Tziaferi ${ }^{9}$, U. Bhawandeep ${ }^{168}$, K. Uchida ${ }^{106}$, B. Ujvari ${ }^{56}$, K.A. Ulmer ${ }^{28,216}$, R. Ulrich ${ }^{66}$, S. Undleeb ${ }^{218}$, L. Uplegger ${ }^{70}$, E. Usai ${ }^{83}$, L. Uvarov ${ }^{173}$, A. Uzunian ${ }^{208}$, E.W. Vaandering ${ }^{70}$, D. Vadruccio ${ }^{126}$, I. Vai ${ }^{98}$, J. Vaitkus ${ }^{228}$, N. Valls ${ }^{158}$, V. Valuev ${ }^{32}$, A. Van Spilbeeck ${ }^{6}$, G.P. Van Onsem ${ }^{231}$, H. Van Haevermaet ${ }^{6}$, I. Van Parijs ${ }^{231}$, M. Van De Klundert ${ }^{6}$, N. Van Remortel ${ }^{6}$, P. Van Mulders ${ }^{231}$, P. Van Mechelen ${ }^{6}$, P. Van Hove ${ }^{178}$, W. Van Doninck ${ }^{231}$, W. Van Driessche ${ }^{81}$, C. Vander Velde ${ }^{129}$, M. Vander Donckt ${ }^{131}$, L. Vanelderen ${ }^{83}$, A. Vanhoefer ${ }^{83}$, S. Vanini ${ }^{97,167}$, P. Vanlaer ${ }^{129}$, I. Vardanyan ${ }^{202}$, F.I. Vardarl1 ${ }^{113}$, J. Varela ${ }^{126}$, N. Varelas ${ }^{105}$, M. Varma ${ }^{135}$, A. Vartak ${ }^{34}$, S. Vavilov ${ }^{173}$, F. Vazquez Valencia ${ }^{104}$, M. Vazquez Acosta ${ }^{8,106}$, C. Veelken ${ }^{149}$, M. Velasco ${ }^{157}$, D. Velicanu ${ }^{135}$, J. Velkovska ${ }^{226}$, R. Venditti ${ }^{90,13}$, S. Ventura ${ }^{97}$, A. Venturi ${ }^{100}$, P. Verdier ${ }^{131}$, P.G. Verdini ${ }^{100}$, G.I. Veres ${ }^{60,28}$, M. Vergili ${ }^{53}$, T. Verlage ${ }^{184}$, C. Vernieri ${ }^{70}$, P. Verwilligen ${ }^{90}, \mathrm{M}$. Verzetti ${ }^{189}, \mathrm{M}$. Verzocchi ${ }^{70}$, V. Veszpremi ${ }^{234}$, G. Vesztergombi ${ }^{60,234}$, J. Veverka $^{135}$, M. Vidal Marono ${ }^{39}$, R. Vidal $^{70}$, I. Vila ${ }^{72}$, R. Vilar Cortabitarte ${ }^{72}$, A. Vilela Pereira ${ }^{62}$, L. Viliani ${ }^{28,93,71}$, T. Virdee ${ }^{106}$, S. Viret ${ }^{131}$, P. Vischia ${ }^{126}$, P. Vitulo ${ }^{98,171}$, J.M. Vizan Garcia ${ }^{164}$, E. Vlasov ${ }^{219}$, J.R. Vlimant ${ }^{31}$, H. Vogel ${ }^{37}$, A. Volkov ${ }^{208}$, I. Volobouev ${ }^{218}$, B. Vormwald ${ }^{83}$, I. Vorobiev ${ }^{37}$, A. Vorobyev ${ }^{173}$, M. Voutilainen ${ }^{58}$, M. Vutova ${ }^{161}$, S.R. Wagner ${ }^{50}$, J. Wagner-Kuhr ${ }^{66}$, M. Walczak ${ }^{65}$, G. Walia ${ }^{168}$, M. Walker ${ }^{193}$, R. Wallny ${ }^{169}$, R. Walsh ${ }^{59}$, W. Waltenberger ${ }^{88}$, W.A.T. Wan Abdullah ${ }^{146}$, C. Wang ${ }^{87}$, D. Wang ${ }^{207}$, F. Wang ${ }^{183}$, J. Wang ${ }^{135}$, J. Wang ${ }^{73}$, Q. Wang ${ }^{119}$, R.-J. Wang ${ }^{156}$, S. Wang ${ }^{73}$, T.W. Wang ${ }^{135}$, Y. Wang ${ }^{229}$, Z. Wang ${ }^{87}$, N. Wardle ${ }^{28}$, 
S. Wasserbaech ${ }^{225,34}$, S. Wayand ${ }^{66}$, M. Wayne ${ }^{158}$, H. Weber ${ }^{184}$, H.A. Weber ${ }^{70}$, M. Weber ${ }^{66}$, M. Weber ${ }^{32}$, H. Wei ${ }^{33}$, T. Weiler ${ }^{66}$, M. Weinberg ${ }^{76}$, C. Welke ${ }^{34}$, L. Wendland ${ }^{85}$, Y. Weng ${ }^{52}$, C. West ${ }^{35}$, J. Wetzel ${ }^{111}$, A. Whitbeck ${ }^{70}$, N. Wickramage ${ }^{192,212}$, S. Wilbur ${ }^{30}$, T. Williams ${ }^{194}$, S. Wimpenny ${ }^{33}$, B.L. Winer ${ }^{163}$, C. Wissing ${ }^{59}$, P. Wittich ${ }^{52}$, B. Wittmer ${ }^{184}$, H.K. Wöhri ${ }^{28}$, C. Wöhrmann ${ }^{66}$, M. Wolf ${ }^{158}$, R. Wolf ${ }^{66}$, E. Wolfe ${ }^{229}$, D. Wood ${ }^{156}$, J. Wood ${ }^{229}$, A. Woodard ${ }^{158}$, N. Woods ${ }^{235}$, S.D. Worm ${ }^{194}$, D. Wright ${ }^{128}$, Z. Wu ${ }^{105}$, H.W. Wulsin ${ }^{163}$, C.-E. Wulz ${ }^{227,88}$, F. Würthwein ${ }^{34}$, B. Wyslouch ${ }^{135}$, F. Xia ${ }^{229}$, M. Xiao ${ }^{115}$, S. Xie ${ }^{31}$, W. Xie ${ }^{183}$, Y. Xin ${ }^{115}$, L. Xu ${ }^{183}$, Q. Xu ${ }^{226}$, Z. Xu ${ }^{207}$, A. Yagil ${ }^{34}$, M. Yalvac ${ }^{137}$, F. Yang ${ }^{70}$, M. Yang ${ }^{135}$, Y. Yang ${ }^{224}$, E. Yazgan ${ }^{81}$, J. Yelton ${ }^{73}$, E.A. Yetkin ${ }^{139,20}$, T. Yetkin ${ }^{237,20}$, K. Yi ${ }^{111}$, Y. Yilmaz ${ }^{124}$, R. Yohay ${ }^{30}$, R. Yonamine ${ }^{129}$, H.D. Yoo ${ }^{200}$, J. Yoo ${ }^{35}$, A. York ${ }^{215}$, C. You ${ }^{115}$, I. Yu ${ }^{211}$, S.S. Yu ${ }^{144}$, F. Yumiceva ${ }^{74}$, M.N. Yusli ${ }^{146}$, J. Zabel ${ }^{188}$, A. Zabi ${ }^{124}$, N. Zaganidis ${ }^{81}$, A. Zagozdzinska ${ }^{232,28}$, M. Zakaria ${ }^{105}$, P. Zalewski ${ }^{145}$, A. Zanetti ${ }^{103}$, M. Zanetti ${ }^{97,167,221}$, A. Zarubin ${ }^{116}$, M. Zeinali ${ }^{187}$, F. Zenoni ${ }^{129}$, S.C. Zenz ${ }^{106}$, W.D. Zeuner ${ }^{28}$, G. Zevi Della Porta ${ }^{34}$, M. Zeyrek ${ }^{137}$, A. Zghiche ${ }^{55}$, F. Zhang ${ }^{207,129}$, H. Zhang ${ }^{87}$, J. Zhang ${ }^{156}$, A. Zhokin ${ }^{219}$, R.Y. Zhu ${ }^{31}$, V. Zhukov ${ }^{202,184}$, V. Zhukova ${ }^{135}$, C. Zorbilmez ${ }^{53}$, P. Zotto ${ }^{97,167}$, D. Zou ${ }^{22}$, A.J. Zsigmond ${ }^{234}$, A. Zucchetta ${ }^{28,97,167}$, A. Zuranski ${ }^{180}$, D. d'Enterria ${ }^{28}$, J.F. de Trocóniz ${ }^{11}$, P. de Barbaro ${ }^{189}$, T. du Pree ${ }^{28}$

${ }^{1}$ Academy of Scientific Research and Technology of the Arab Republic of Egypt, Egyptian Network of High Energy Physics, Cairo, Egypt

2 Adiyaman University, Adiyaman, Turkey

3 Ain Shams University, Cairo, Egypt

${ }^{4}$ The University of Alabama, Tuscaloosa, USA

5 Albert Einstein Center for Fundamental Physics, Bern, Switzerland

${ }^{6}$ Universiteit Antwerpen, Antwerpen, Belgium

${ }^{7}$ Argonne National Laboratory, Argonne, USA

${ }^{8}$ Instituto de Astrofísica de Canarias, La Laguna, Spain

${ }^{9}$ University of Athens, Athens, Greece

10 University of Auckland, Auckland, New Zealand

11 Universidad Autónoma de Madrid, Madrid, Spain

12 Universidad Autónoma de San Luis Potosí, San Luis Potosí, Mexico 13 Università di Bari, Bari, Italy

${ }^{14}$ Università della Basilicata, Potenza, Italy 15 Baylor University, Waco, USA

16 University of Belgrade, Faculty of Physics and Vinca Institute of Nuclear Sciences, Belgrade, Serbia

17 Benemerita Universidad Autonoma de Puebla, Puebla, Mexico

18 Beni-Suef University, Bani Sweif, Egypt

19 Bhabha Atomic Research Centre, Mumbai, India

${ }^{20}$ Bogazici University, Istanbul, Turkey

21 Università di Bologna, Bologna, Italy 


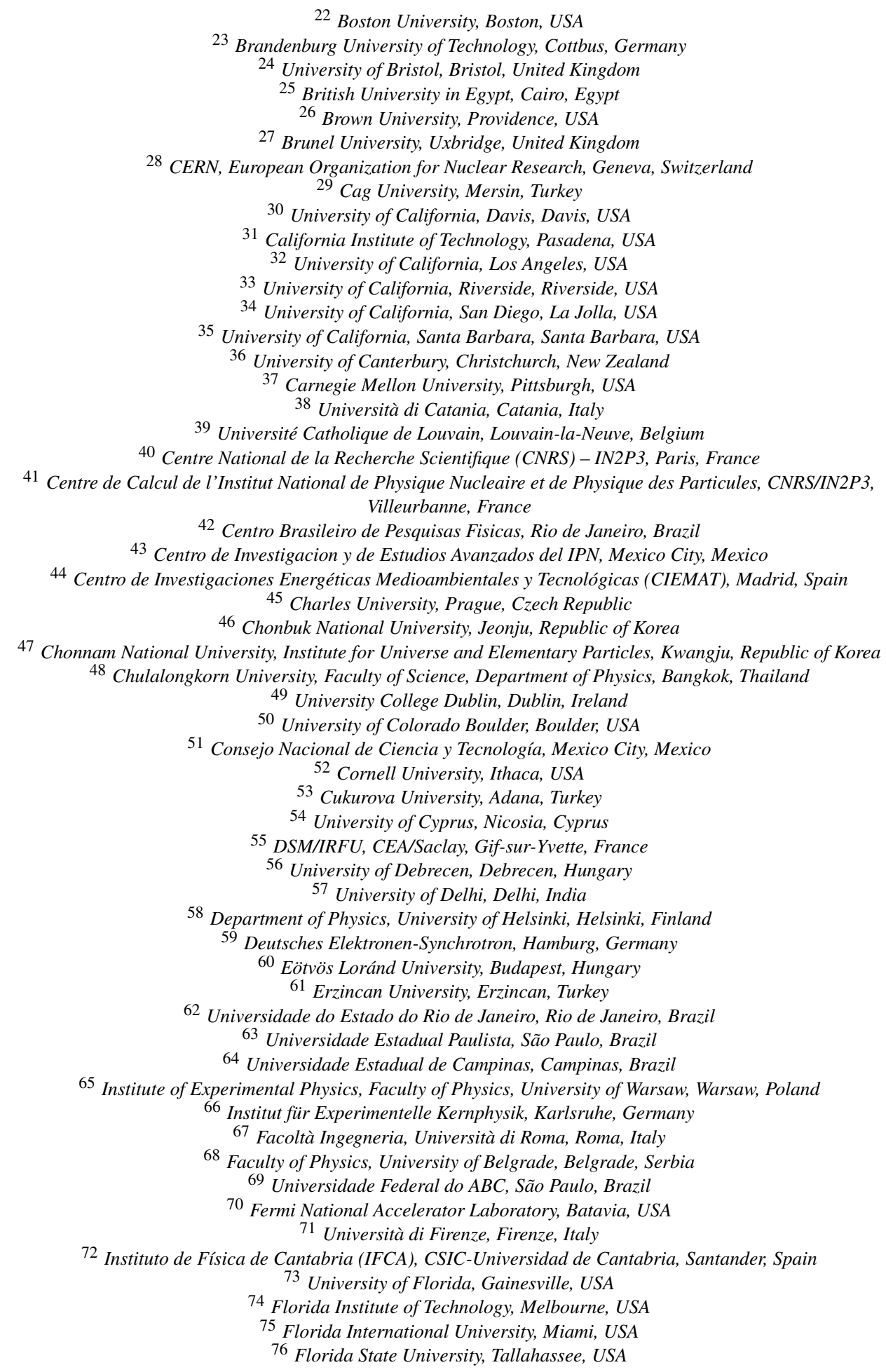


77 Università G. Marconi, Roma, Italy

78 Gaziosmanpasa University, Tokat, Turkey

${ }^{79}$ Università di Genova, Genova, Italy

80 Georgian Technical University, Tbilisi, Georgia

81 Ghent University, Ghent, Belgium

82 Hacettepe University, Ankara, Turkey

83 University of Hamburg, Hamburg, Germany

84 Université de Haute Alsace, Mulhouse, France

85 Helsinki Institute of Physics, Helsinki, Finland

${ }^{86}$ Helwan University, Cairo, Egypt

87 Institute of High Energy Physics, Beijing, China

88 Institut für Hochenergiephysik der OeAW, Wien, Austria

${ }^{89}$ INFN Laboratori Nazionali di Frascati, Frascati, Italy

${ }^{90}$ INFN Sezione di Bari, Bari, Italy

91 INFN Sezione di Bologna, Bologna, Italy

92 INFN Sezione di Catania, Catania, Italy

93 INFN Sezione di Firenze, Firenze, Italy

94 INFN Sezione di Genova, Genova, Italy

95 INFN Sezione di Milano-Bicocca, Milano, Italy

96 INFN Sezione di Napoli, Napoli, Italy

97 INFN Sezione di Padova, Padova, Italy

98 INFN Sezione di Pavia, Pavia, Italy

99 INFN Sezione di Perugia, Perugia, Italy

100 INFN Sezione di Pisa, Pisa, Italy

101 INFN Sezione di Roma, Roma, Italy

102 INFN Sezione di Torino, Torino, Italy

103 INFN Sezione di Trieste, Trieste, Italy

104 Universidad Iberoamericana, Mexico City, Mexico

105 University of Illinois at Chicago (UIC), Chicago, USA

106 Imperial College, London, United Kingdom

107 Indian Institute of Science Education and Research, Bhopal, India

108 Indian Institute of Science Education and Research (IISER), Pune, India

109 International Islamic University of Malaysia, Kuala Lumpur, Malaysia

110 University of Ioánnina, Ioánnina, Greece

111 The University of Iowa, Iowa City, USA

112 Isfahan University of Technology, Isfahan, Iran

113 Istanbul Technical University, Istanbul, Turkey

114 Izmir Institute of Technology, Izmir, Turkey

115 Johns Hopkins University, Baltimore, USA

116 Joint Institute for Nuclear Research, Dubna, Russia

117 Kafkas University, Kars, Turkey

118 Kangwon National University, Chunchon, Republic of Korea

119 The University of Kansas, Lawrence, USA

${ }^{120}$ Kansas State University, Manhattan, USA

${ }^{121}$ King Abdulaziz University, Jeddah, Saudi Arabia

122 Korea University, Seoul, Republic of Korea

123 Kyungpook National University, Daegu, Republic of Korea

${ }^{124}$ Laboratoire Leprince-Ringuet, Ecole Polytechnique, IN2P3-CNRS, Palaiseau, France

125 Laboratori Nazionali di Legnaro dell'INFN, Legnaro, Italy

126 Laboratório de Instrumentação e Física Experimental de Partículas, Lisboa, Portugal

127 Lappeenranta University of Technology, Lappeenranta, Finland

128 Lawrence Livermore National Laboratory, Livermore, USA

129 Université Libre de Bruxelles, Bruxelles, Belgium

130 Universidad de Los Andes, Bogota, Colombia

131 Université de Lyon, Université Claude Bernard Lyon 1, CNRS-IN2P3, Institut de Physique Nucléaire de Lyon, Villeurbanne, France 
132 Malaysian Nuclear Agency, MOSTI, Kajang, Malaysia

133 Marmara University, Istanbul, Turkey

134 University of Maryland, College Park, USA

135 Massachusetts Institute of Technology, Cambridge, USA 136 Mersin University, Mersin, Turkey

137 Middle East Technical University, Physics Department, Ankara, Turkey

138 Università di Milano-Bicocca, Milano, Italy

139 Mimar Sinan University, Istanbul, Istanbul, Turkey

140 University of Minnesota, Minneapolis, USA

141 University of Mississippi, Oxford, USA

142 Université de Mons, Mons, Belgium

143 Università di Napoli 'Federico II', Napoli, Italy

144 National Central University, Chung-Li, Taiwan

145 National Centre for Nuclear Research, Swierk, Poland

146 National Centre for Particle Physics, Universiti Malaya, Kuala Lumpur, Malaysia

147 National Centre for Particle and High Energy Physics, Minsk, Belarus

148 National Centre for Physics, Quaid-I-Azam University, Islamabad, Pakistan

149 National Institute of Chemical Physics and Biophysics, Tallinn, Estonia

${ }^{150}$ National Institute of Science Education and Research, Bhubaneswar, India

151 National Research Nuclear University 'Moscow Engineering Physics Institute' (MEPhI), Moscow, Russia

152 National Scientific Center, Kharkov Institute of Physics and Technology, Kharkov, Ukraine

153 National Taiwan University (NTU), Taipei, Taiwan

154 National Technical University of Athens, Athens, Greece

155 University of Nebraska-Lincoln, Lincoln, USA

156 Northeastern University, Boston, USA

157 Northwestern University, Evanston, USA

158 University of Notre Dame, Notre Dame, USA

159 Institute of Nuclear Research ATOMKI, Debrecen, Hungary

160 Institute for Nuclear Research, Moscow, Russia

161 Institute for Nuclear Research and Nuclear Energy, Sofia, Bulgaria

162 Institute of Nuclear and Particle Physics (INPP), NCSR Demokritos, Aghia Paraskevi, Greece

163 The Ohio State University, Columbus, USA

164 Universidad de Oviedo, Oviedo, Spain

165 Ozyegin University, Istanbul, Turkey

166 P.N. Lebedev Physical Institute, Moscow, Russia

167 Università di Padova, Padova, Italy

168 Panjab University, Chandigarh, India

169 Institute for Particle Physics, ETH Zurich, Zurich, Switzerland

${ }^{170}$ Paul Scherrer Institut, Villigen, Switzerland

171 Università di Pavia, Pavia, Italy

172 Università di Perugia, Perugia, Italy

173 Petersburg Nuclear Physics Institute, Gatchina (St. Petersburg), Russia

174 Università del Piemonte Orientale, Novara, Italy

175 Piri Reis University, Istanbul, Turkey

176 Università di Pisa, Pisa, Italy

177 Plasma Physics Research Center, Science and Research Branch, Islamic Azad University, Tehran, Iran

178 Institut Pluridisciplinaire Hubert Curien, Université de Strasbourg, Université de Haute Alsace Mulhouse, CNRS/IN2P3, Strasbourg, France

179 Politecnico di Bari, Bari, Italy

180 Princeton University, Princeton, USA

181 University of Puerto Rico, Mayaguez, USA

182 Purdue University Calumet, Hammond, USA

183 Purdue University, West Lafayette, USA

${ }^{184}$ RWTH Aachen University, I. Physikalisches Institut, Aachen, Germany

185 RWTH Aachen University, III. Physikalisches Institut A, Aachen, Germany

186 RWTH Aachen University, III. Physikalisches Institut B, Aachen, Germany 
187 Institute for Research in Fundamental Sciences (IPM), Tehran, Iran

188 Rice University, Houston, USA

189 University of Rochester, Rochester, USA

190 Università di Roma, Roma, Italy

191 Institute Rudjer Boskovic, Zagreb, Croatia

192 University of Ruhuna, Matara, Sri Lanka

193 Rutgers, The State University of New Jersey, Piscataway, USA

${ }^{194}$ Rutherford Appleton Laboratory, Didcot, United Kingdom

195 Saha Institute of Nuclear Physics, Kolkata, India

196 School of Physics and Astronomy, University of Southampton, Southampton, United Kingdom

197 Institute for Scintillation Materials of National Academy of Science of Ukraine, Kharkov, Ukraine

198 Scuola Normale Superiore di Pisa, Pisa, Italy

199 Scuola Normale e Sezione dell'INFN, Pisa, Italy

200 Seoul National University, Seoul, Republic of Korea

201 University of Seoul, Seoul, Republic of Korea

202 Skobeltsyn Institute of Nuclear Physics, Lomonosov Moscow State University, Moscow, Russia 203 University of Sofia, Sofia, Bulgaria

204 University of Split, Faculty of Electrical Engineering, Mechanical Engineering and Naval Architecture, Split, Croatia

205 University of Split, Faculty of Science, Split, Croatia

206 St. Petersburg State Polytechnical University, St. Petersburg, Russia

207 State Key Laboratory of Nuclear Physics and Technology, Peking University, Beijing, China

208 State Research Center of Russian Federation, Institute for High Energy Physics, Protvino, Russia

209 State University of New York at Buffalo, Buffalo, USA

210 Università degli Studi di Siena, Siena, Italy

211 Sungkyunkwan University, Suwon, Republic of Korea

212 Tata Institute of Fundamental Research, Mumbai, India

213 Tbilisi State University, Tbilisi, Georgia

214 University of Tehran, Department of Engineering Science, Tehran, Iran

215 University of Tennessee, Knoxville, USA

216 Texas A\&M University, College Station, USA

217 Texas A\&M University at Qatar, Doha, Qatar

218 Texas Tech University, Lubbock, USA

219 Institute for Theoretical and Experimental Physics, Moscow, Russia

220 Università di Torino, Torino, Italy

221 Università di Trento, Trento, Italy

222 Università di Trento (Trento), Padova, Italy

223 Università di Trieste, Trieste, Italy

224 Universität Zürich, Zurich, Switzerland

225 Utah Valley University, Orem, USA

226 Vanderbilt University, Nashville, USA

227 Vienna University of Technology, Vienna, Austria

228 Vilnius University, Vilnius, Lithuania

229 University of Virginia, Charlottesville, USA

230 University of Visva-Bharati, Santiniketan, India

231 Vrije Universiteit Brussel, Brussel, Belgium

232 Warsaw University of Technology, Institute of Electronic Systems, Warsaw, Poland

233 Wayne State University, Detroit, USA

234 Wigner Research Centre for Physics, Budapest, Hungary

235 University of Wisconsin, Madison, USA

236 Yerevan Physics Institute, Yerevan, Armenia

237 Yildiz Technical University, Istanbul, Turkey

238 Zewail City of Science and Technology, Zewail, Egypt

* Deceased. 